\title{
The minimal operator and the geometric maximal operator in $\mathbb{R}^{n}$
}

\author{
by \\ David Cruz-URibe, SFO (Hartford, CT)
}

Abstract. We prove two-weight norm inequalities in $\mathbb{R}^{n}$ for the minimal operator

$$
m f(x)=\inf _{Q \ni x} \frac{1}{|Q|} \int_{Q}|f| d y,
$$

extending to higher dimensions results obtained by Cruz-Uribe, Neugebauer and Olesen [8] on the real line. As an application we extend to $\mathbb{R}^{n}$ weighted norm inequalities for the geometric maximal operator

$$
M_{0} f(x)=\sup _{Q \ni x} \exp \left(\frac{1}{|Q|} \int_{Q} \log |f| d x\right),
$$

proved by Yin and Muckenhoupt [27].

We also give norm inequalities for the centered minimal operator, study powers of doubling weights and give sufficient conditions for the geometric maximal operator to be equal to the closely related limiting operator $M_{0}^{*} f=\lim _{r \rightarrow 0} M\left(|f|^{r}\right)^{1 / r}$.

\section{Introduction}

1.1. The minimal operator. Given a measurable function $f$, the minimal function of $f, m f$, is defined by

$$
m f(x)=\inf _{Q \ni x} \frac{1}{|Q|} \int_{Q}|f| d y,
$$

where the supremum is taken over all cubes $Q$ whose sides are parallel to the co-ordinate axes. By the Lebesgue differentiation theorem, $m f(x) \leq$ $|f(x)| \leq M f(x)$ almost everywhere; intuitively, the minimal operator controls where $f$ is small, just as the Hardy-Littlewood maximal operator controls where $f$ is large.

2000 Mathematics Subject Classification: Primary 42B25.

Key words and phrases: minimal operator, geometric maximal operator, weighted norm inequalities.

I want to thank Carlo Sbordone for his hospitality and the graduate students at the 1997 Trimester on Variational Problems for their thought-provoking suggestions. Both contributed greatly to the completion of this paper. 
The minimal operator was introduced by Cruz-Uribe and Neugebauer [5], who used it to study the fine structure of $A_{p}$ weights. They also considered the weighted norm inequalities for the minimal operator. This question is complicated by the fact that if $f \in L^{p}\left(\mathbb{R}^{n}\right)$ then $m f(x) \equiv 0$. One solution is to take inverses, making something small (the minimal operator) into something large (which can be measured by a norm inequality). In the oneweight case they proved the following result. Here and below by a weight we mean a non-negative, locally integrable function.

TheOrem 1.1. Given a weight $w$, the following are equivalent:

(i) $w \in A_{\infty}$;

(ii) there exists a constant $C$ such that for every $p, 0<p<\infty$, and every $t>0$,

$$
w\left(\left\{x \in \mathbb{R}^{n}: m f(x)<1 / t\right\}\right) \leq \frac{C}{t^{p}} \int_{\mathbb{R}^{n}} \frac{w}{|f|^{p}} d x
$$

for every $f$ such that $1 / f \in L^{p}(w)$;

(iii) there exists a constant $C$ such that for every $p, 0<p<\infty$,

$$
\int_{\mathbb{R}^{n}} \frac{w}{(m f)^{p}} d x \leq C \int_{\mathbb{R}^{n}} \frac{w}{|f|^{p}} d x
$$

for every $f$ such that $1 / f \in L^{p}(w)$.

Theorem 1.1 follows from Hölder's inequality and the one-weight norm inequalities for the maximal operator. Two-weight norm inequalities are significantly more difficult: working on the real line, Cruz-Uribe, Neugebauer and Olesen [8] were able to prove the following result.

Theorem 1.2. Given a pair of weights $(u, v)$ and $p, 0<p<\infty$, let $\sigma=v^{1 /(p+1)}$. Then the following are equivalent:

(i) $(u, v) \in W_{p}$ : there exists a constant $C_{1}$ such that for every interval $I$,

$$
\frac{1}{|I|} \int_{I} u d x \leq C_{1}\left(\frac{1}{|I|} \int_{I} \sigma d x\right)^{p+1} ;
$$

(ii) there exists a constant $C_{2}$ such that for every $t>0$,

$$
u(\{x \in \mathbb{R}: m f(x)<1 / t\}) \leq \frac{C_{2}}{t^{p}} \int_{\mathbb{R}} \frac{v}{|f|^{p}} d x
$$

for every $f$ such that $1 / f \in L^{p}(v)$;

(iii) $(u, v) \in W_{p}^{*}$ : there exists a constant $C_{3}$ such that for every interval I,

$$
\int_{I} \frac{u}{m\left(\sigma / \chi_{I}\right)^{p}} d x \leq C_{3} \int_{I} \sigma d x
$$


(iv) there exists a constant $C_{4}$ such that

$$
\int_{\mathbb{R}} \frac{u}{(m f)^{p}} d x \leq C_{4} \int_{\mathbb{R}} \frac{v}{|f|^{p}} d x
$$

for every $f$ such that $1 / f \in L^{p}(v)$.

Remarks. (i) In Theorem 1.2, the constants $C_{2}$ and $C_{4}$ depend on $C_{1}$ and $C_{3}$, respectively, but do not depend on $p$. However, $C_{3} \approx p C_{1}$.

(ii) Unlike the Hardy-Littlewood maximal operator, the strong and weak-type norm inequalities for the minimal operator are governed by the same pairs of weights.

(iii) In the one-weight case the $W_{p}$ condition becomes a reverse Hölder inequality which is equivalent to the $A_{\infty}$ condition. See [5] for details.

The proof of Theorem 1.2 in [8] depends heavily on the special covering lemmas for the real line, and does not extend in a satisfactory manner to higher dimensions. In particular, to prove the weak-type norm inequality in $\mathbb{R}^{n}$ we had to assume that $u$ was a doubling weight, but to prove the strong-type norm inequality we had to assume (among other things) that $\sigma=v^{1 /(p+1)}$ was a doubling weight.

The main result of this paper is an extension of Theorem 1.2 to $\mathbb{R}^{n}$, $n>1$, with uniform assumptions on $u$ and $v$.

TheOREm 1.3. Given $p, 0<p<\infty$, let $(u, v)$ be a pair of weights such that either $u$ or $\sigma=v^{1 /(p+1)}$ is a doubling weight. Then in $\mathbb{R}^{n}, n>1$, conditions (i)-(iv) of Theorem 1.2 are equivalent, with intervals replaced by cubes in (i) and (iii).

The relation between the constants $C_{i}, 1 \leq i \leq 4$, depends on whether $u$ or $\sigma$ is doubling. Let $B_{n}$ be the constant in the Besicovitch-Morse covering lemma (see Lemma 2.1 below). If $u$ is a doubling weight with constant $D(u)$ then

$$
\begin{aligned}
& C_{2} \leq B_{n} D(u) C_{1}, \\
& C_{3} \leq C_{1}+p B_{n} 2^{-n} D(u) C_{1}, \\
& C_{4} \leq 8 D(u)^{2} C_{3}+8 \cdot 3^{n} p B_{n} D(u) C_{3} .
\end{aligned}
$$

If $\sigma$ is a doubling weight with constant $D(\sigma)$ then

$$
\begin{aligned}
& C_{2} \leq 2^{-n p} B_{n} D(\sigma)^{p+1} C_{1}, \\
& C_{3} \leq C_{1}+p B_{n} 2^{-n(p+1)} D(\sigma)^{p+1} C_{1}, \\
& C_{4} \leq 8 D(\sigma)^{2} C_{3} .
\end{aligned}
$$

1.2. The geometric maximal operator. As an application of Theorem 1.3 we prove two-weight norm inequalities in $\mathbb{R}^{n}$ for the geometric maximal 
operator

$$
M_{0} f(x)=\sup _{Q \ni x} \exp \left(\frac{1}{|Q|} \int_{Q} \log |f| d x\right),
$$

and the closely related limiting operator

$$
M_{0}^{*} f(x)=\lim _{r \rightarrow 0} M_{r} f(x)=\lim _{r \rightarrow 0} M\left(|f|^{r}\right)(x)^{1 / r} .
$$

Norm inequalities for the geometric maximal operator on the real line were studied by Yin and Muckenhoupt [27] and others. Cruz-Uribe and Neugebauer [6] used Theorem 1.2 to give a new proof of their results and to prove analogous results for $M_{0}^{*}$. A key step in their proof is showing that for a dense family of functions $f$,

$$
M_{0} f(x)=\lim _{r \rightarrow 0} m\left(|f|^{-r}\right)(x)^{-1 / r}, \quad x \in \mathbb{R}^{n} .
$$

They used this to derive norm inequalities for the geometric maximal operator from norm inequalities for the minimal operator via a limiting argument. This was possible since the constants $C_{2}$ and $C_{4}$ in Theorem 1.2 are independent of $p$. (See [6] for details of the proof and for the history of the problem.)

Using Theorem 1.3 we can immediately extend their results to higher dimensions - their proofs go through without change.

THEOREM 1.4. Given a pair of weights $(u, v)$, suppose that either $u$ is doubling or $\sigma_{q}=v^{1 /(q+1)}$ is doubling for all $q$ sufficiently large and

$$
\limsup _{q \rightarrow \infty} 2^{-n q} D\left(\sigma_{q}\right)^{q+1}<\infty .
$$

Then the following are equivalent:

(i) $(u, v) \in W_{\infty}$ : there exists a constant $C$ such that for every cube $Q$,

$$
\frac{1}{|Q|} \int_{Q} u d x \leq C \exp \left(\frac{1}{|Q|} \int_{Q} \log v d x\right) ;
$$

(ii) there exists a constant $C$ such that for all $p, 0<p<\infty$,

$$
u\left(\left\{x \in \mathbb{R}^{n}: M_{0} f(x)>t\right\}\right) \leq \frac{C}{t^{p}} \int_{\mathbb{R}^{n}}|f|^{p} v d x
$$

for every $f \in L^{p}(v)$.

TheOREM 1.5. Given a pair of weights $(u, v)$, suppose that $\sigma_{q}=v^{1 /(q+1)}$ is doubling for all $q$ sufficiently large and

$$
\limsup _{q \rightarrow \infty} D\left(\sigma_{q}\right)<\infty .
$$

Then the following are equivalent: 
(i) $(u, v) \in W_{\infty}^{*}$ : there exists a constant $C$ such that for all cubes $Q$,

$$
\int_{Q} M_{0}\left(v^{-1} \chi_{Q}\right) u d x \leq C|Q|
$$

(ii) there exists a constant $C$ such that for all $p, 0<p<\infty$,

$$
\int_{\mathbb{R}^{n}}\left(M_{0} f\right)^{p} u d x \leq C \int_{\mathbb{R}^{n}}|f|^{p} v d x
$$

for every $f \in L^{p}(v)$.

TheOREM 1.6. Theorems 1.4 and 1.5 remain true if $M_{0}$ is everywhere replaced by $M_{0}^{*}$, provided that $v \in I_{\infty}$ :

$$
\limsup _{Q, \sigma} \frac{1}{|Q|}\left(\frac{1}{|Q|} \int_{Q} v^{-\sigma} d x\right)^{1 / \sigma}<\infty
$$

where the upper limit is taken over all cubes $Q$ containing the origin and all $\sigma>0$ as $|Q|$ tends to infinity and $\sigma$ tends to 0 . The $I_{\infty}$ condition is necessary as well as sufficient.

REMARKS. (i) Unlike the minimal operator, the two-weight, weak and strong-type norm inequalities for the geometric maximal operator are governed by different weight classes. See Yin and Muckenhoupt [27] or CruzUribe and Neugebauer [6] for an example. We suspect that this is connected to the fact that doubling conditions on $u$ are sufficient in Theorem 1.4 but not in Theorem 1.5, but we are uncertain as to what the exact relation is.

(ii) A serendipitous consequence of our work on the geometric maximal operator was the discovery that Theorem 1.3 could be re-interpreted as a result about a maximal operator. Define the harmonic maximal operator by

$$
M_{-1} f(x)=\sup _{Q \ni x}\left(\frac{1}{|Q|} \int_{Q}|f|^{-1} d y\right)^{-1} ;
$$

in other words, $M_{-1} f(x)$ is the supremum of the harmonic averages of $f$ on cubes containing $x$. It is immediate that

$$
M_{-1} f(x)=m\left(f^{-1}\right)(x)^{-1},
$$

and Theorem 1.3 can be restated in terms of $M_{-1}$.

1.3. Doubling conditions. While the doubling conditions we assume in Theorems 1.3-1.6 are not unreasonable (cf. Wheeden [23]) they are not necessary: in Example 5.8 below we construct a pair $(u, v) \in W_{p}$ such that neither $u$ nor $v$ is doubling, but the weak and strong-type inequalities hold. However, we have repeatedly and unsuccessfully attempted either to prove Theorem 1.3 without doubling conditions or to construct a non-doubling pair 
$(u, v)$ which satisfy the $W_{p}$ condition but for which the norm inequalities do not hold. This question remains open.

As a corollary to our proofs, we can show that the strong $W_{p}$ condition,

$$
\frac{1}{|2 Q|} \int_{2 Q} u d x \leq C_{1}^{\prime}\left(\frac{1}{|Q|} \int_{Q} \sigma d x\right)^{p+1},
$$

and the strong $W_{p}^{*}$ condition,

$$
\int_{3 Q} \frac{u}{m\left(\sigma / \chi_{3 Q}\right)^{p}} d x \leq C_{3}^{\prime} \int_{Q} \sigma d x
$$

are equivalent and are sufficient - without additional doubling conditionsfor the weak and strong-type norm inequalities for the minimal operator to hold. However, Example 5.8 below shows that they are not necessary.

The strong $W_{p}$ condition also yields a sufficient condition for Theorem 1.4 and the corresponding part of Theorem 1.6 to hold:

$$
\text { strong } W_{\infty}: \quad \frac{1}{|2 Q|} \int_{2 Q} u d x \leq C \exp \left(\frac{1}{|Q|} \int_{Q} \log v d x\right) .
$$

Surprisingly, the strong $W_{p}^{*}$ condition does not yield a satisfactory sufficient condition for Theorem 1.5 to hold. The best we can obtain is a Sawyer-type condition involving a related but larger maximal operator.

1.4. The centered minimal operator. Another approach to eliminating doubling conditions is to modify the operator by restricting the collection of cubes over which it is defined. For example, while the weighted maximal operator, $M_{w}$, is not necessarily bounded in higher dimensions unless $w$ is doubling (see Sjögren [20]), the weighted centered maximal operator and the weighted dyadic maximal operator are for all weights $w$.

The proof of Theorem 1.3 below or the proof of Theorem 1.2 in [8] are readily adapted to the dyadic minimal operator. The results in [6] also carry over to the dyadic geometric maximal operator with little change. Details are left to the reader.

The centered minimal operator, $m_{c}$ (defined as in equation (1.1) but with the infimum restricted to cubes centered at $x$ ), is both more interesting and more difficult. As was noted in [8], unlike the maximal operator, the minimal operator and the centered minimal operator are not equivalent. A simple example on the real line is given by $e^{x}: m\left(e^{x}\right) \equiv 0$ but $m_{c}\left(e^{x}\right)=e^{x}$. In [8] it was conjectured that a "centered $W_{p}$ " condition is necessary and sufficient for the weak-type inequality for $m_{c}$. Here we adapt the proof of Theorem 1.3 to $m_{c}$ and show that this condition (and the corresponding Sawyer-type condition) are necessary and sufficient for both the strong and weak-type norm inequalities. 
Theorem 1.7. Given weights $(u, v)$ and $p, 0<p<\infty$, let $\sigma=v^{1 /(p+1)}$. Then the following are equivalent:

(i) $(u, v) \in W_{p, c}$ : there exists a constant $D_{1}$ such that for every cube $Q$,

$$
\frac{1}{|Q|} \int_{Q} u d x \leq D_{1}\left(\frac{1}{|2 Q|} \int_{2 Q} \sigma d x\right)^{p+1} ;
$$

(ii) there exists a constant $D_{2}$ such that for every $t>0$,

$$
u\left(\left\{x \in \mathbb{R}^{n}: m_{c} f(x)<1 / t\right\}\right) \leq \frac{D_{2}}{t^{p}} \int_{\mathbb{R}^{n}} \frac{v}{|f|^{p}} d x
$$

for every $f$ such that $1 / f \in L^{p}(v)$;

(iii) $(u, v) \in W_{p, c}^{*}$ : there exists a constant $D_{3}$ such that for every cube $Q$,

$$
\int_{Q} \frac{u}{m_{c}\left(\sigma / \chi_{Q}\right)^{p}} d x \leq D_{3} \int_{Q} \sigma d x
$$

(iv) there exists a constant $D_{4}$ such that

$$
\int_{\mathbb{R}^{n}} \frac{u}{\left(m_{c} f\right)^{p}} d x \leq D_{4} \int_{\mathbb{R}^{n}} \frac{v}{|f|^{p}} d x
$$

for every $f$ such that $1 / f \in L^{p}(v)$.

Remarks. (i) Unfortunately, except in the case $n=1$ (for which case we have a special proof) the constants $D_{2}$ and $D_{4}$ are not independent of $p$, so we cannot use the limiting technique of Cruz-Uribe and Neugebauer to prove the analogues of Theorems 1.4-1.6 for the centered geometric maximal operator, $M_{0, c}$. Even in the case $n=1$ the proof breaks down, since the Sawyer-type condition

$$
W_{\infty, c}^{*}: \quad \int_{Q} M_{0, c}\left(v^{-1} \chi_{Q}\right) u d x \leq C|Q|
$$

does not seem to imply the $W_{p, c}^{*}$ condition. The question of weighted norm inequalities for the centered geometric maximal operator and their relationship to norm inequalities for the centered minimal operator remains open.

(ii) We do not have control of the constant $D_{2}$ since for the centered minimal operator we do not have a direct proof that the $W_{p, c}$ condition implies the weak-type inequality. It would be very interesting to have such a proof, as it may yield a proof of a weak-type inequality for $M_{0, c}$.

1.5. Organization. The remainder of this paper is organized as follows:

In Sections 2-4 we prove Theorem 1.3. In Section 2 we show that the $W_{p}$ condition is equivalent to the weak-type inequality; in Section 3 we show that the $W_{p}$ and $W_{p}^{*}$ conditions are the same; and in Section 4 we show that the $W_{p}^{*}$ condition is equivalent to the strong-type inequality. At the end of 
each section we derive from the proofs the non-doubling sufficient conditions for the minimal operator and geometric maximal operator discussed above.

In Section 5 we examine the doubling conditions imposed on $v$ in Theorems 1.3-1.5. We give examples of weights for which they do hold, and construct an example to show that there exists a doubling weight $v$ such that $v^{r}$ is not doubling for any $r, 0<r<1$. This answers in the negative questions posed in Cruz-Uribe [3, p. 561] and Cruz-Uribe, Neugebauer and Olesen [8]. We also construct a pair of functions $(u, v)$ in $\mathbb{R}^{n}$ which satisfy the $W_{p}$ condition, are not doubling, and for which the strong-type norm inequality for $m$ holds.

In Section 6 we prove Theorem 1.7. The key to the proof is a slightly stronger version of the Besicovitch-Morse covering lemma. Further, we prove an analogue of Theorem 1.1 by showing that in the one-weight case the $W_{p, c}$ condition is equivalent to a weak $A_{\infty}$ condition due to Sawyer [19]: given any cube $Q$ and a measurable set $E \subset Q$, there exist constants $C$ and $\delta$ such that

$$
\text { weak } A_{\infty}: \quad \frac{w(E)}{w(2 Q)} \leq C\left(\frac{|E|}{|Q|}\right)^{\delta} .
$$

Finally, in Section 7 we consider a problem first studied in detail by Cruz-Uribe and Neugebauer [6]: sufficient conditions on a function $f$ for the equality $M_{0} f(x)=M_{0}^{*} f(x)$ to hold. They showed that this equality does not hold in general, but showed that if $\log f$ is locally integrable and $f \in L^{p}$ for some $p>0$ then it holds almost everywhere. We generalize this result considerably.

Theorem 1.8. Let $v \in I_{\infty}$. Suppose $f \in L^{p}(v)$ for some $p>0$ and suppose there exists a cube $Q_{0}$ (possibly infinite) such that $\operatorname{supp} f=Q_{0}$ and $\log f \in L_{\mathrm{loc}}^{1}\left(Q_{0}\right)$. Then $M_{0} f(x)=M_{0}^{*} f(x)$ almost everywhere. Further, equality almost everywhere is the best possible.

Theorem 1.8 is a partial generalization of a recent result by Wik [26], and we discuss the relation between the two results. Also, as a corollary to Theorem 1.8 we show that the best constants in the one-weight, strong-type norm inequalities for $M_{0}$ and for the limiting operator $M_{0}^{*}$ are the same. This proves a conjecture made by Wik.

Throughout this paper all notation is standard or will be defined as needed. For the convenience of the reader, the principal definitions will be repeated in the relevant sections. All cubes are assumed to have their sides parallel to the co-ordinate axes. Given a cube $Q, l(Q)$ will denote the length of its sides and for any $r>0, r Q$ will denote the cube with the same center as $Q$ and such that $l(r Q)=r l(Q)$. By weights we will always mean nonnegative, locally integrable functions which are positive on a set of positive 
measure. Given a Lebesgue measurable set $E$ and a weight $w,|E|$ will denote the Lebesgue measure of $E, w(E)=\int_{E} w d x$ and $w / \chi_{E}$ will denote the function equal to $w$ on $E$ and infinity elsewhere. We say that a non-negative measure $\mu$ is a doubling measure if for any cube $Q, \mu(2 Q) \leq D(\mu) \mu(Q) ; D(\mu)$ is called the doubling constant of $\mu$. If $\mu$ satisfies the doubling condition for all $Q$ such that $2 Q \subset Q_{0}$, we say that $\mu$ is a doubling measure on $Q_{0}$. If $\mu$ is doubling and $d \mu=w d x$, we say that $w$ is a doubling weight. Given $1<p<\infty, p^{\prime}=p /(p-1)$ will denote the conjugate exponent of $p$. Finally, $C$ will denote a positive constant whose value may change at each appearance.

2. The weak-type norm inequality. In this section we prove that the $W_{p}$ condition,

$$
\frac{1}{|Q|} \int_{Q} u d x \leq C_{1}\left(\frac{1}{|Q|} \int_{Q} \sigma d x\right)^{p+1},
$$

is equivalent to the weak-type inequality for the minimal operator in $\mathbb{R}^{n}$. The proof requires the Besicovitch-Morse covering lemma. We state the precise version we need; for a proof see de Guzmán [13].

Lemma 2.1. Given a bounded set $E$ in $\mathbb{R}^{n}$, suppose that for each $x \in E$ there exists a cube $Q_{x}$ such that $x \in \frac{1}{2} Q_{x}$. Then there exists a sequence $\left\{Q_{k}\right\}$ of $Q_{x}$ 's such that $E$ is contained in the union of the $Q_{k}$ 's; further, there exists a constant $B_{n}$, depending only on the dimension $n$, such that every point of $\mathbb{R}^{n}$ is contained in at most $B_{n}$ of the $Q_{k}$ 's.

Throughout this section, let $\sigma=v^{1 /(p+1)}$.

To show that the weak-type norm inequality implies (2.1), fix a cube $Q$ and let $f=\sigma \chi_{Q}$. If $\sigma(Q)=\infty$ then (2.1) is immediate. If it is finite then for each $x \in Q$,

$$
m f(x) \leq \frac{1}{|Q|} \int_{Q} \sigma d x .
$$

If we let $1 / t$ in the weak-type inequality equal the right-hand side we get

$$
u(Q) \leq C_{2}\left(\frac{1}{|Q|} \int_{Q} \sigma d x\right)^{p} \cdot \int_{Q} \sigma d x,
$$

and this is the $W_{p}$ condition.

To prove that (2.1) implies the weak-type inequality, fix a function $f$ such that $1 / f \in L^{p}(v)$. Without loss of generality we may assume that $f$ is non-negative. Further, we first assume that there exists a cube $P$ such that $f$ is positive on $P$ and $\operatorname{supp}(1 / f)=P$. 
Fix $t>0$ and let $E_{t}=\left\{x \in \mathbb{R}^{n}: m f(x)<1 / t\right\}$. Then for every $x \in E_{t}$ there exists a cube $Q_{x}$ containing $x$ such that

$$
\frac{1}{\left|Q_{x}\right|} \int_{Q_{x}} f d y<1 / t
$$

Each $Q_{x}$ is contained in the cube $P$ and $E_{t}$ is contained in their union. Therefore, by Lemma 2.1, there exists a sequence $\left\{Q_{j}\right\}$ of $Q_{x}$ 's such that $\bigcup_{j} 2 Q_{j}$ also contains $E_{t}$ and such that the $Q_{j}$ 's have overlap of at most $B_{n}$.

Since $u$ or $\sigma$ is doubling, (2.1) implies the strong $W_{p}$ condition (1.2); if $u$ is doubling then $C_{1}^{\prime}=2^{-n} D(u) C_{1}$; otherwise, if $\sigma$ is doubling then $C_{1}^{\prime}=2^{-n(p+1)} D(\sigma)^{p+1} C_{1}$. Therefore,

$$
u\left(E_{t}\right) \leq \sum_{j} u\left(2 Q_{j}\right) \leq 2^{n} C_{1}^{\prime} \sum_{j}\left|Q_{j}\right|^{-p}\left(\int_{Q_{j}} \sigma d x\right)^{p+1} .
$$

By Hölder's inequality, for each $j$,

$$
\int_{Q_{j}} \sigma d x \leq\left(\int_{Q_{j}} \frac{v}{f^{p}} d x\right)^{1 /(p+1)}\left(\int_{Q_{j}} f d x\right)^{p /(p+1)} .
$$

Hence, since $Q_{j}=Q_{x}$ for some $x \in E_{t}$,

$$
\begin{aligned}
2^{n} C_{1}^{\prime} \sum_{j}\left|Q_{j}\right|^{-p}\left(\int_{Q_{j}} \sigma d x\right)^{p+1} & \leq \frac{2^{n} C_{1}^{\prime}}{t^{p}} \sum_{j}\left(\int_{Q_{j}} \sigma d x\right)^{p+1}\left(\int_{Q_{j}} f d x\right)^{-p} \\
& \leq \frac{2^{n} C_{1}^{\prime}}{t^{p}} \sum_{j} \int_{Q_{j}} \frac{v}{f^{p}} d x \\
& \leq \frac{2^{n} B_{n} C_{1}^{\prime}}{t^{p}} \int_{\mathbb{R}^{n}} \frac{v}{f^{p}} d x
\end{aligned}
$$

This implies that the weak-type inequality holds with $C_{2}=2^{n} B_{n} C_{1}^{\prime}$.

To complete the proof, fix any non-negative $f \in L^{p}(v)$ and define the sequence of functions $f_{k}=(f+1 / k) / \chi_{P_{k}}$, where $P_{k}$ is the cube centered at the origin with $l\left(P_{k}\right)=k$. Then each $f_{k}$ is strictly positive on $P_{k}$, $\operatorname{supp}\left(1 / f_{k}\right)=P_{k}$ and $1 / f_{k} \in L^{p}(v)$ since $1 / f \in L^{p}(v)$. The sequence $\left\{f_{k}\right\}$ decreases to $f$, so the sequence $\left\{m f_{k}\right\}$ also decreases and $\lim _{n \rightarrow \infty} m f_{k} \geq m f$. On the other hand, for a fixed $x \in \mathbb{R}^{n}$ and any $\varepsilon>0$, there exists a cube $Q$ such that for all $k$ sufficiently large, $Q \subset P_{k}$ and

$$
m f(x) \geq \frac{1}{|Q|} \int_{Q} f d y-\varepsilon \geq m f_{k}(x)-\varepsilon .
$$

Therefore, $\left\{m f_{k}\right\}$ converges to $m f$, and by the monotone convergence theorem the strong-type inequality holds for all $f$. 
REMARK. Even without a doubling condition, the $W_{p}$ condition is necessary for the weak-type inequality to hold. In the proof that it is sufficient, we only used a doubling condition to prove that the strong $W_{p}$ condition holds. Hence the strong $W_{p}$ condition is itself a sufficient condition which does not involve doubling. However, Example 5.8 shows that this condition is not necessary. By Jensen's inequality, if $(u, v)$ satisfy the strong $W_{\infty}$ condition (1.4) then $(u, v)$ satisfy the strong $W_{p}$ condition for all $p>0$ with a constant independent of $p$. This is enough to show that the strong $W_{\infty}$ condition is sufficient for the weak-type norm inequalities for $M_{0}$ and $M_{0}^{*}$ to hold (for the latter provided $v \in I_{\infty}$ ). See [6] for details.

3. The equivalence of $W_{p}$ and $W_{p}^{*}$. In this section we prove that the $W_{p}$ condition,

$$
\frac{1}{|Q|} \int_{Q} u d x \leq C_{1}\left(\frac{1}{|Q|} \int_{Q} \sigma d x\right)^{p+1},
$$

and the $W_{p}^{*}$ condition,

$$
\int_{Q} \frac{u}{m\left(\sigma / \chi_{Q}\right)^{p}} d x \leq C_{3} \int_{Q} \sigma d x
$$

are equivalent. Throughout this section, let $\sigma=v^{1 /(p+1)}$.

We first show that (3.2) implies (3.1). Fix a cube $Q$. If $\sigma(Q)=\infty$ then the $W_{p}$ condition holds trivially, so assume it is finite. Then for every $x \in Q, m\left(\sigma / \chi_{Q}\right)(x) \leq \sigma(Q)$. If $\sigma(Q)=0$ then the left-hand side of the $W_{p}^{*}$ condition is finite only if $u(Q)=0$, and again the $W_{p}$ condition holds trivially. But if $\sigma(Q)>0$ then we can substitute this inequality into (3.2) and (3.1) follows immediately.

To prove that (3.1) implies (3.2), fix a cube $Q$. We may assume without loss of generality that $u(Q)>0$ and $\sigma(Q)<\infty$, since otherwise the $W_{p}^{*}$ condition holds trivially. For each $t>0$ let $E_{t}=\left\{x \in Q: m\left(\sigma / \chi_{Q}\right)(x)<\right.$ $1 / t\}$. Then

$$
\int_{Q} \frac{u}{m\left(\sigma / \chi_{Q}\right)^{p}} d x=p \int_{0}^{R} t^{p-1} u\left(E_{t}\right) d t+p \int_{R}^{\infty} t^{p-1} u\left(E_{t}\right) d t,
$$

where $R$ will be chosen below. Since $u\left(E_{t}\right) \leq u(Q)$, the first integral on the right-hand side is bounded by $u(Q) R^{p}$.

To estimate the second integral on the right-hand side of (3.3) we proceed as in the proof of the weak-type inequality. Given $t>0$, for each $x \in E_{t}$ there exists a cube $Q_{x}^{t} \subset Q$ containing $x$ such that

$$
\frac{1}{\left|Q_{x}^{t}\right|} \int_{Q_{x}^{t}} \sigma d x<1 / t
$$


The cubes $2 Q_{x}^{t}$ cover $E_{t}$, so by Lemma 2.1 there exists a sequence of cubes $\left\{Q_{j}^{t}\right\}$ which have finite overlap and such that the cubes $2 Q_{j}^{t}$ still cover $E_{t}$. If we combine this with the strong $W_{p}$ condition (1.2) (which holds since $u$ or $\sigma$ is doubling) and inequality (3.4), it follows that

$$
\begin{aligned}
p \int_{R}^{\infty} t^{p-1} u\left(E_{t}\right) d t & \leq p \int_{R}^{\infty} t^{p-1} \sum_{j} u\left(2 Q_{j}^{t}\right) d t \\
& \leq 2^{n} p C_{1}^{\prime} \int_{R}^{\infty} t^{p-1} \sum_{j}\left|Q_{j}^{t}\right|\left(\frac{1}{\left|Q_{j}^{t}\right|} \int_{Q_{j}^{t}} \sigma d x\right)^{p+1} \\
& \leq 2^{n} p C_{1}^{\prime} \int_{R}^{\infty} t^{-2} \sum_{j}\left|Q_{j}^{t}\right| d t \\
& \leq 2^{n} p C_{1}^{\prime} B_{n} \int_{R}^{\infty} t^{-2}\left|E_{t}\right| d t r \leq 2^{n} p C_{1}^{\prime} B_{n} R^{-1}|Q| .
\end{aligned}
$$

Therefore,

$$
\int_{Q} \frac{u}{m\left(\sigma / \chi_{Q}\right)^{p}} d x \leq u(Q) R^{p}+2^{n} p C_{1}^{\prime} B_{n} R^{-1}|Q| .
$$

Let $R=|Q| / \sigma(Q)$. Then by $(3.1), u(Q) R^{p} \leq C_{1} \sigma(Q)$. Hence

$$
\int_{Q} \frac{u}{m\left(\sigma / \chi_{Q}\right)^{p}} d x \leq\left(C_{1}+2^{n} p C_{1}^{\prime} B_{n}\right) \sigma(Q)
$$

which is (3.2) with $C_{3}=C_{1}+2^{n} p C_{1}^{\prime} B_{n}$.

REMARK. As with the weak-type inequality in Section 2, we only used a doubling condition to get the strong $W_{p}$ condition (1.2). Notice, however, that $C_{3}$ contains a factor of $p$. Because of this, even though the weak and strong-type norm inequalities for the minimal operator are both governed by the same class of weights $-W_{p}$-this is no longer the case for the geometric maximal operator.

4. The strong-type norm inequality. In this section we complete the proof of Theorem 1.3 by showing that the $W_{p}^{*}$ condition,

$$
\int_{Q} \frac{u}{m\left(\sigma / \chi_{Q}\right)^{p}} d x \leq C_{3} \int_{Q} \sigma d x,
$$

is equivalent to the strong-type norm inequality for the minimal operator in $\mathbb{R}^{n}$. Throughout this section, let $\sigma=v^{1 /(p+1)}$.

The proof that the strong-type inequality implies (4.1) is essentially the same as the proof in Section 2 that the weak-type inequality implies the 
$W_{p}$ condition: fix a cube $Q$, let $f=\sigma / \chi_{Q}$ and substitute this into the strong-type inequality. The $W_{p}^{*}$ condition follows at once.

The proof that (4.1) implies the strong-type inequality is based on the proof of the strong-type norm inequality for the maximal operator given by Sawyer [18], and adapts ideas from Cruz-Uribe [4], Cruz-Uribe and Neugebauer [7] and Cruz-Uribe, Neugebauer and Olesen [9] to higher dimensions. For the proof we need two lemmas.

Lemma 4.1. If $(u, v) \in W_{p}^{*}$ and either $u$ or $\sigma$ is doubling, then the strong $W_{p}^{*}$ condition (1.3) holds. If $u$ is doubling then $C_{3}^{\prime} \leq D(u)^{2} C_{1}+6^{n} p C_{1}^{\prime} B_{n}$; if $\sigma$ is doubling then $C_{3}^{\prime} \leq D(\sigma)^{2} C_{3}$.

Proof. If $\sigma$ is doubling then the strong $W_{p}^{*}$ condition follows immediately from (4.1).

If $u$ is doubling, then, as shown in Section $3,(u, v) \in W_{p}$ with constant $C_{1} \leq C_{3}$. We now repeat the proof that the $W_{p}$ condition implies the $W_{p}^{*}$ condition, except that we replace $Q$ with $3 Q$. Then inequality (3.6) becomes

$$
\begin{aligned}
\int_{3 Q} \frac{u}{m\left(\sigma / \chi_{3 Q}\right)^{p}} d x & \leq u(3 Q) R^{p}+2^{n} p C_{1}^{\prime} B_{n} R^{-1}|3 Q| \\
& \leq D(u)^{2} u(Q) R^{p}+6^{n} p C_{1}^{\prime} B_{n} R^{-1}|Q| .
\end{aligned}
$$

The rest of the proof now goes through as before to yield the desired inequality with $C_{3}^{\prime}=D(u)^{2} C_{1}+6^{n} p C_{1}^{\prime} B_{n}$.

The second lemma is a classical covering theorem. For the convenience of the reader we sketch the proof.

Lemma 4.2. Given a finite collection $\left\{Q_{i}\right\}_{i=1}^{N}$ of cubes, there exists a disjoint subcollection $\left\{Q_{i_{j}}\right\}_{j=1}^{k}$ such that each cube $Q_{r}$ is contained in $3 Q_{i_{s}}$ for some s.

Proof. We may assume that the cubes are ordered so that $l\left(Q_{1}\right) \geq \ldots \geq$ $l\left(Q_{N}\right)$. Let $i_{1}=1$. If any cube intersects $Q_{i_{1}}$ then it is contained in $3 Q_{i_{1}}$. Let $Q_{i_{2}}$ be the largest cube which is disjoint from $Q_{i_{1}}$. Repeating this argument we form the desired subcollection.

To prove that (4.1) implies the strong-type inequality, we first assume that $v$ is everywhere positive; we treat the general case at the end. Fix a function $f$ such that $1 / f \in L^{p}(v)$. Without loss of generality we may assume that $f$ is non-negative.

Fix $\alpha>1$. For each integer $k$, let $A_{k}=\left\{x \in \mathbb{R}^{n}: \alpha^{-(k+1)} \leq m f(x)\right.$ $\left.<\alpha^{-k}\right\}$. If $x \in A_{k}$ then there exists a cube $Q_{x}^{k}$ containing $x$ such that

$$
\alpha^{-(k+1)} \leq \frac{1}{\left|Q_{x}^{k}\right|} \int_{Q_{x}^{k}} f d y<\alpha^{-k} .
$$


By the continuity of the integral we may choose each $Q_{x}^{k}$ so that the coordinates of its center and $l\left(Q_{x}^{k}\right)$ are rational. But then the set $\left\{Q_{x}^{k}\right\}_{x \in A_{k}}$ is countable, so we may enumerate its elements as $\left\{Q_{j}^{k}\right\}$. Clearly $A_{k}$ is contained in their union. By induction define the sequence of disjoint sets $E_{j}^{k}$ : $E_{1}^{k}=Q_{1}^{k} \cap A_{k}, \quad E_{2}^{k}=\left(Q_{2}^{k} \cap A_{k}\right) \backslash E_{1}^{k}, \quad E_{3}^{k}=\left(Q_{3}^{k} \cap A_{k}\right) \backslash\left(E_{1}^{k} \cup E_{2}^{k}\right), \ldots$ Then $A_{k}=\bigcup_{j} E_{j}^{k}$, and since the $A_{k}$ 's are disjoint, the $E_{j}^{k}$ 's are pairwise disoint for all $j$ and $k$.

As shown in Section 3 , since $(u, v) \in W_{p}^{*},(u, v) \in W_{p}$. So by the weaktype inequality, $u(\{x: m f(x)=0\})=0$. Therefore,

$$
\begin{aligned}
\int_{\mathbb{R}^{n}} \frac{u}{(m f)^{p}} d x & =\sum_{k} \int_{A_{k}} \frac{u}{(m f)^{p}} d x \leq \sum_{k} u\left(A_{k}\right) \alpha^{p(k+1)} \\
& \leq \alpha^{p} \sum_{j, k} u\left(E_{j}^{k}\right)\left(\frac{1}{\left|Q_{j}^{k}\right|} \int_{Q_{j}^{k}} f d x\right)^{-p} .
\end{aligned}
$$

Since $v$ is positive, $\sigma\left(Q_{j}^{k}\right)>0$. Further, by Hölder's inequality,

$$
\begin{aligned}
\sigma\left(Q_{j}^{k}\right) & \leq\left(\int_{\mathbb{R}^{n}} \frac{v}{f^{p}} d x\right)^{1 /(p+1)}\left(\int_{Q_{j}^{k}} f d x\right)^{p /(p+1)} \\
& \leq\left(\int_{\mathbb{R}^{n}} \frac{v}{f^{p}} d x\right)^{1 /(p+1)}\left(\alpha^{-k}\left|Q_{j}^{k}\right|\right)^{p /(p+1)}<\infty .
\end{aligned}
$$

Therefore,

$$
\begin{aligned}
\alpha^{p} \sum_{j, k} u\left(E_{j}^{k}\right) & \left(\frac{1}{\left|Q_{j}^{k}\right|} \int_{Q_{j}^{k}} f d x\right)^{-p} \\
& =\alpha^{p} \sum_{j, k} u\left(E_{j}^{k}\right)\left(\frac{1}{\left|Q_{j}^{k}\right|} \int_{Q_{j}^{k}} \sigma d x\right)^{-p}\left(\frac{\int_{Q_{j}^{k}}(f / \sigma) \cdot \sigma d x}{\int_{Q_{j}^{k}} \sigma d x}\right)^{-p} .
\end{aligned}
$$

Let $X=\mathbb{N} \times \mathbb{Z}$ and define the measure $\omega$ on $X$ by

$$
\omega(j, k)=u\left(E_{j}^{k}\right)\left(\frac{1}{\left|Q_{j}^{k}\right|} \int_{Q_{j}^{k}} \sigma d x\right)^{-p} .
$$

Given a non-negative, measurable function $h$, define the operators $S$ and $T$ by

$$
\operatorname{Sh}(j, k)=\frac{\int_{Q_{j}^{k}} \sigma d x}{\int_{Q_{j}^{k}} h \sigma d x} \quad \text { and } \quad \operatorname{Th}(j, k)=\frac{\int_{Q_{j}^{k}} h \sigma d x}{\int_{Q_{j}^{k}} \sigma d x} .
$$

By Hölder's inequality, for any $r>1$,

$$
\operatorname{Sh}(j, k) \leq T\left(h^{1-r^{\prime}}\right)(j, k)^{r-1} .
$$


Set $r=1+2 / p$; then we may rewrite the above inequality as

$$
\int_{\mathbb{R}^{n}} \frac{u}{(m f)^{p}} d x \leq \alpha^{p} \int_{X} S(f / \sigma)^{p} d \omega \leq \alpha^{p} \int_{X} T\left((f / \sigma)^{-p / 2}\right)^{2} d \omega .
$$

If $T$ were a bounded operator from $L^{2}(\sigma)$ to $L^{2}(X, \omega)$ then

$$
\int_{\mathbb{R}^{n}} \frac{u}{(m f)^{p}} d x \leq \alpha^{p} C \int_{\mathbb{R}^{n}} \frac{\sigma^{p}}{f^{p}} \sigma d x=\alpha^{p} C \int_{\mathbb{R}^{n}} \frac{v}{f^{p}} d x .
$$

Since $T$ is bounded on $L^{\infty}$ with constant 1 , by the Marcinkiewicz interpolation theorem it will suffice to show that $T$ is weak $(1,1)$. For each $\lambda>0$, let

$$
E_{\lambda}=\{(j, k) \in X: T h(j, k)>\lambda\}
$$

Then

$$
\omega\left(E_{\lambda}\right)=\sum_{(j, k) \in E_{\lambda}} u\left(E_{j}^{k}\right)\left(\frac{1}{\left|Q_{j}^{k}\right|} \int_{Q_{j}^{k}} \sigma d x\right)^{-p} \leq \sum_{(j, k) \in E_{\lambda}} \int_{E_{j}^{k}} \frac{u}{m\left(\sigma / \chi_{Q_{j}^{k}}\right)^{p}} d x .
$$

For each $M>0$, let $E_{M}=\left\{(j, k) \in E_{\lambda}: j+|k| \leq M\right\}$. Then it will suffice to show that there is a constant $C$ independent of $M$ such that

$$
\sum_{(j, k) \in E_{M}} \int_{E_{j}^{k}} \frac{u}{m\left(\sigma / \chi_{Q_{j}^{k}}\right)^{p}} d x \leq \frac{C}{\lambda} \int_{\mathbb{R}^{n}} h \sigma d x .
$$

Since the set $\left\{Q_{j}^{k}:(j, k) \in E_{M}\right\}$ is finite, by Lemma 4.2 there exists a disjoint subcollection $\left\{Q_{n}\right\}$ such that each cube $Q_{j}^{k} \subset 3 Q_{n}$ for some $n$. Therefore, since the $E_{j}^{k}$ 's are pairwise disjoint and $E_{j}^{k} \subset Q_{j}^{k}$,

$$
\begin{aligned}
\sum_{(j, k) \in E_{M}} \int_{E_{j}^{k}} \frac{u}{m\left(\sigma / \chi_{Q_{j}^{k}}\right)^{p}} d x & \leq \sum_{n} \sum_{Q_{j}^{k} \subset 3 Q_{n}} \int_{E_{j}^{k}} \frac{u}{m\left(\sigma / \chi_{3 Q_{n}}\right)^{p}} d x \\
& \leq \sum_{n} \int_{3 Q_{n}} \frac{u}{m\left(\sigma / \chi_{\left.3 Q_{n}\right)^{p}}\right.} d x \\
& \leq C_{3}^{\prime} \sum_{n} \int_{Q_{n}} \sigma d x
\end{aligned}
$$

The last inequality follows from Lemma 4.1. Since each $Q_{n}=Q_{j}^{k}$ for some $(j, k) \in E_{\lambda}$, and since the $Q_{n}$ 's are disjoint,

$$
C_{3}^{\prime} \sum_{n} \int_{Q_{n}} \sigma d x \leq \frac{C_{3}^{\prime}}{\lambda} \sum_{n} \int_{Q_{n}} h \sigma d x \leq \frac{C_{3}^{\prime}}{\lambda} \int_{\mathbb{R}^{n}} h \sigma d x .
$$

Hence $T$ is weak-type $(1,1)$ with constant $C_{3}^{\prime}$. Therefore, $T$ is strong-type $(2,2)$ with constant $8 C_{3}^{\prime}$ (see, for example, Sadosky [17]), and so the strongtype inequality for the minimal operator holds for positive $v$ with constant 
$C_{4} \leq 8 \alpha^{p} C_{3}^{\prime}$. However, there are no restrictions on $\alpha>1$, so we may take the limit as $\alpha$ tends to 1 to get $C_{4} \leq 8 C_{3}^{\prime}$.

To complete the proof, fix an arbitrary pair $(u, v) \in W_{p}^{*}$ and fix any nonnegative $f$ such that $1 / f \in L^{p}(v)$. For each $k>0$ define $v_{k}=v+k^{-(n+p+1)}$ and $f_{k}=(f+1 / k) / \chi_{P_{k}}$, where $P_{k}$ is the cube centered at the origin with $l\left(P_{k}\right)=k$. Then a calculation shows that $\left(u, v_{k}\right) \in W_{p}^{*}$ with the same constant as $(u, v)$. Further, $1 / f_{k} \in L^{p}\left(v_{k}\right)$. As we showed at the end of Section $2, m f_{k}$ decreases to $m f$. Therefore, by the special case given above,

$$
\begin{aligned}
\int_{\mathbb{R}^{n}} \frac{u}{\left(m f_{k}\right)^{p}} d x & \leq C_{4} \int_{P_{k}} \frac{v_{k}}{f_{k}^{p}} d x \\
& \leq C_{4} \int_{\mathbb{R}^{n}} \frac{v}{f^{p}} d x+C_{4} k^{-(n+p+1)} k^{p}\left|P_{k}\right| .
\end{aligned}
$$

The strong-type norm inequality now follows from the monotone convergence theorem.

REMARK. Even without a doubling condition the $W_{p}^{*}$ condition is necessary for the strong-type inequality to hold. In the proof that it is sufficient, we only used a doubling condition to prove Lemma 4.1, so the strong $W_{p}^{*}$ condition (1.3) is a sufficient condition which does not use doubling. The proof of Lemma 4.1 also shows that the strong $W_{p}$ condition implies the strong $W_{p}^{*}$ condition. Hence, combining this observation with the remarks at the end of Section 2, we see that the strong $W_{p}$ condition is actually sufficient for both the weak and strong-type inequalities.

However, even though we were able to use the strong $W_{p}$ condition to show that the strong $W_{\infty}$ condition is a sufficient condition for the weak-type norm inequality for the geometric maximal operator, a similar argument fails to produce a sufficient condition for the strong-type inequality for $M_{0}$. To intuitively see why, note that while the strong $W_{\infty}$ condition is the (formal) limit of the strong $W_{p}$ condition as $p$ tends to infinity, the (formal) limit of the strong $W_{p}^{*}$ condition is

$$
\int_{3 Q} M_{0}\left(v^{-1} \chi_{3 Q}\right) u d x \leq C|Q| .
$$

Since $|3 Q|=3^{n}|Q|$, this is equivalent to the $W_{\infty}^{*}$ condition, and so nothing new is gained.

However, by introducing a larger maximal operator, we get a Sawyertype condition which is sufficient. We define the "offset" geometric maximal operator by

$$
M_{0}^{o} f(x)=\sup _{3 Q \ni x} \exp \left(\frac{1}{|Q|} \int_{Q} \log f d x\right) .
$$


Then following the argument in [6], if $(u, v)$ are such that for all cubes $Q$,

$$
\int_{Q} M_{0}^{o}\left(v^{-1} \chi_{Q}\right) u d x \leq C|Q|,
$$

then $(u, v)$ satisfy the strong $W_{p}^{*}$ condition for all $p$ with a constant independent of $p$. Hence this yields a sufficient condition for the strong-type norm inequality for $M_{0}$ that does not require a doubling condition, but one of limited utility.

5. Doubling conditions on $v^{r}, 0<r<1$. In this section we examine the doubling conditions which appear in Theorems 1.3, 1.4 and 1.5. These results assume different but closely related doubling conditions on a weight $v$ :

(D1) for all $r, 0<r<1, v^{r}$ is a doubling weight;

(D2) for all $r$ sufficiently small, $v^{r}$ is a doubling weight and

$$
\limsup _{r \rightarrow 0} D\left(v^{r}\right)<\infty
$$

(D3) for all $r$ sufficiently small, $v^{r}$ is a doubling weight and

$$
\limsup _{r \rightarrow 0} 2^{-n / r} D\left(v^{r}\right)^{1 / r}<\infty .
$$

Condition (D1) is equivalent to saying that $v=w^{1 / r}$, where $w$ is a doubling weight. Note that this does not imply that $v$ is a doubling weight: a simple counter-example on the real line is $v(x)=\min \left(1,|x|^{-1}\right)$.

Examples of doubling weights are well known: for instance, if $w \in A_{\infty}$ then $w$ is a doubling weight. Examples of doubling weights which are not in $A_{\infty}$ have been constructed by Fefferman and Muckenhoupt [10], Strömberg [22] and Wik [25]. However, it is not obvious a priori that there exist weights which satisfy conditions (D2) and (D3). We begin by showing that the $A_{\infty}$ condition implies both.

Lemma 5.1. If $w \in A_{\infty}$ then conditions (D1)-(D3) hold.

Proof. By carefully keeping track of constants we can show $w^{r}$ is doubling for all $r<1$ and that condition (D3) holds. (It is immediate that (D3) implies (D2).)

Fix $w \in A_{\infty}$; then $w \in A_{p}$ for some $p>1$ with constant $A_{p}(w)$. Then, given any cube $Q$ and $r<1$, by Hölder's inequality,

$$
\begin{aligned}
\int_{2 Q} w^{r} d x & \leq|2 Q|\left(\frac{1}{|2 Q|} \int_{2 Q} w d x\right)^{r} \\
& \leq 2^{n} A_{p}(w)^{r}|Q|\left(\frac{1}{|2 Q|} \int_{2 Q} w^{1-p^{\prime}} d x\right)^{-r(p-1)}
\end{aligned}
$$




$$
\begin{aligned}
& \leq 2^{n+n r(p-1)} A_{p}(w)^{r}|Q|\left(\frac{1}{|Q|} \int_{Q}\left(w^{r}\right)^{\left(1-p^{\prime}\right) / r} d x\right)^{-r(p-1)} \\
& \leq 2^{n+n r(p-1)} A_{p}(w)^{r} \int_{Q} w^{r} d x
\end{aligned}
$$

Hence $D\left(w^{r}\right) \leq 2^{n+n r(p-1)} A_{p}(w)^{r}$ and condition (D3) follows immediately.

The situation for doubling weights which are not in $A_{\infty}$ is more complex. We will construct the following two examples. They are similar in spirit to the one of Fefferman and Muckenhoupt; we use an idea of Wik's to show they are not in $A_{\infty}$.

EXAMPLE 5.2. There exists a weight $v$ on $\mathbb{R}$ such that $v$ is a doubling weight but for any $r>0, r \neq 1, v^{r}$ is not a doubling weight.

EXAMPLE 5.3. There exists a weight $w$ on $\mathbb{R}$ such that for every $r>0$ $w^{r}$ is a doubling weight and $D\left(w^{r}\right)$ is uniformly bounded for all $r \leq 1$, but $w^{r}$ is not in $A_{\infty}$.

We believe that Example 5.3 also satisfies condition (D3) but we cannot get a sufficiently sharp estimate on the doubling constant to show this. Further, after repeated attempts we were unable to construct a weight for which (D2) holds but (D3) does not. Thus the following questions remain open:

(i) Does there exist a weight $v$ and $0<a<b$ such that $v^{r}$ is doubling for $a \leq r \leq b$, but not for $r<a$ or $r>b$ ?

(ii) Does there exist a weight $v$ such that $v^{r}$ is doubling for all $r \leq 1$ but condition (D2) does not hold?

(iii) Are conditions (D2) and (D3) equivalent?

The basic building block for our examples is given in Theorem 5.5. Hereafter, by a dyadic step function we mean a function of the form $\sum a_{i} \chi_{J_{i}}$, where the $J_{i}$ 's are disjoint dyadic intervals.

Definition 5.4. For $\alpha>1$, define the operator $T_{\alpha}$ on dyadic step functions as follows: given a dyadic interval $I=\left[m 2^{k},(m+1) 2^{k}\right)$, let

$$
I_{i}=\left[m 2^{k}+(i-1) 2^{k-2}, m 2^{k}+i 2^{k-2}\right), \quad 1 \leq i \leq 4,
$$

be the four dyadic subintervals of length $|I| / 4$. Then define

$$
T_{\alpha}\left(\chi_{I}\right)=\chi_{I_{1}}+\alpha \chi_{I_{2}}+\alpha^{-1} \chi_{I_{3}}+\chi_{I_{4}} \text {. }
$$

More generally, if $f=\sum a_{i} \chi_{J_{i}}$ is a dyadic step function, define

$$
T_{\alpha}(f)=\sum a_{i} T_{\alpha}\left(\chi_{J_{i}}\right) \text {. }
$$

ThEOREM 5.5. For $\alpha>1$, define the sequence $\left\{u_{\alpha, n}\right\}$ of functions by induction: $u_{\alpha, 0}=\chi_{[0,1)}$ and $u_{\alpha, n}=T_{\alpha}\left(u_{\alpha, n-1}\right)=T_{\alpha}^{n}\left(u_{\alpha, 0}\right)$. Then: 
(i) for each $\alpha, n$ and $r>0, u_{\alpha, n}^{r}=u_{\alpha^{r}, n}$;

(ii) given a dyadic interval $I \subset[0,1],|I| \geq 4^{-n}$, then $u_{\alpha, n+s}(I)=$ $\gamma_{\alpha}^{s} u_{\alpha, n}(I)$, for all $s \geq 1$, where $\gamma_{\alpha}=1 / 2+\left(\alpha+\alpha^{-1}\right) / 4$;

(iii) for each $n$ and $l,-n \leq l \leq n$,

$$
\left|\left\{x \in[0,1]: u_{\alpha, n}(x)=\alpha^{l}\right\}\right|=4^{-n}\left(\begin{array}{c}
2 n \\
n+l
\end{array}\right) ;
$$

(iv) for each $n, u_{\alpha, n}$ is doubling on $[0,1]$ and $D\left(u_{\alpha, n}\right)$ depends on $\alpha$ but not on $n$;

(v) there does not exist $p>1$ such that for all $n, u_{\alpha, n}$ satisfies the $A_{p}$ condition on $[0,1]$ with uniformly bounded constant.

REMARK. For each $n, u_{\alpha, n}$ is bounded and bounded away from zero, so each $u_{\alpha, n}$ is a doubling weight and satisfies the $A_{\infty}$ condition on $[0,1]$.

To prove Theorem 5.5 we need two lemmas.

LEMMA 5.6. Given a weight $v$, suppose there exists a constant $S(v)$ such that if $I$ and $J$ are adjacent dyadic intervals (whose union need not be a dyadic interval), then $v(I) \leq S(v) v(J)$. Then $v$ is a doubling weight and $D(v) \leq P(S(v))$, where $P$ is a degree 5 polynomial.

Proof. Fix an interval $I$; if $j$ is such that $2^{j}<|I| \leq 2^{j+1}$ then $I$ must contain a dyadic interval $I_{d}$ of length $2^{j-1}$. Since $|2 I| \leq 2^{j+2}, 2 I$ must be contained in the union of 8 adjacent dyadic intervals of length $2^{j-1}$. Then $I_{d}$ must be either the third or fourth interval from the left or the right; repeatedly applying our hypothesis shows that

$$
v(2 I) \leq P(S(v)) v\left(I_{d}\right) \leq P(S(v)) v(I),
$$

where

$$
\begin{aligned}
P(S(v))=\max (1+2 S(v)+ & 2 S(v)^{2}+S(v)^{3}+S(v)^{4}+S(v)^{5} \\
1 & \left.1+2 S(v)+2 S(v)^{2}+2 S(v)^{3}+S(v)^{4}\right) .
\end{aligned}
$$

REMARK. Essentially the same proof shows that if $I_{0}$ is a dyadic interval and the hypothesis holds for all dyadic subintervals of $I_{0}$ then $u$ is doubling on $I_{0}$.

The following result is due to Wik [25].

Lemma 5.7. A weight $w$ is in $A_{p}, p>1$, if and only if there exist constants $q>p$ and $C_{w}>0$ such that, given any cube $Q$ and measurable set $E \subset Q$,

$$
\frac{w(E)}{w(Q)} \geq C_{w}\left(\frac{|E|}{|Q|}\right)^{q} .
$$


Proof of Theorem 5.5. By Definition 5.4,

$$
u_{\alpha, n}=\sum_{i=1}^{4^{n}} a_{i, n} \chi_{J_{i}^{n}},
$$

where the $J_{i}^{n}$ 's are disjoint dyadic intervals, $\left|J_{i}^{n}\right|=4^{-n}$. Denote the four dyadic subintervals of $J_{i}^{n}$ of length $4^{-n-1}$ by $J_{i, j}^{n}, 1 \leq j \leq 4$.

We prove part (i) by induction on $n$. Fix $\alpha$ and $r$. It is immediate that $u_{\alpha^{r}, 0}=u_{\alpha, 0}^{r}$. Now suppose that for some $n$,

Then

$$
u_{\alpha^{r}, n}=u_{\alpha, n}^{r}=\sum a_{i, n}^{r} \chi_{J_{i}^{n}} .
$$

$$
\begin{aligned}
u_{\alpha^{r}, n+1} & =T_{\alpha^{r}}\left(u_{\alpha^{r}, n}\right)=\sum_{i} a_{i, n}^{r} \chi_{J_{i, 1}^{n}}+\alpha^{r} a_{i, n}^{r} \chi_{J_{i, 2}^{n}}+\alpha^{-r} a_{i, n}^{r} \chi_{J_{i, 3}^{n}}+a_{i, n}^{r} \chi_{J_{i, 4}^{n}} \\
& =u_{\alpha, n+1}^{r} .
\end{aligned}
$$

Hence this equality holds for all $n$.

To prove part (ii), fix $\alpha$ and $n$. It will suffice to prove that $u_{\alpha, n+1}(I)=$ $\gamma_{\alpha} u_{\alpha, n}(I)$. There exist $i$ and $k$ such that $I=J_{i}^{n} \cup J_{i+1}^{n} \cup \ldots \cup J_{i+k}^{n}$. Hence,

$$
\begin{aligned}
u_{\alpha, n+1}(I) & =T_{\alpha}\left(u_{\alpha, n}\right)(I)=\sum_{j=0}^{k} \int_{J_{i+j}^{n}} a_{i+j, n} T_{\alpha}\left(\chi_{J_{i+j}^{n}}\right) d x \\
& =\gamma_{\alpha} \sum_{j=0}^{k} a_{i+j, n}\left|J_{i+j}^{n}\right|=\gamma_{\alpha} \sum_{j=0}^{k} u_{\alpha, n}\left(J_{i+j}^{n}\right) \\
& =\gamma_{\alpha} u_{\alpha, n}(I) .
\end{aligned}
$$

We prove part (iii) by induction on $n$. For $n=1$ it is immediate. Now suppose it holds for some $n$ and all $l$. Since $u_{\alpha, n+1}=T_{\alpha}\left(u_{\alpha, n}\right)$, for each $l$, the set $\left\{x \in[0,1]: u_{\alpha, n+1}(x)=\alpha^{l}\right\}$ will consist of three disjoint subsets: $x \in[0,1]$ such that $u_{\alpha, n}(x)=\alpha^{l}$ and whose "height" was unchanged by $T_{\alpha} ; x$ such that $u_{\alpha, n}(x)=\alpha^{l-1}$ and whose height was raised to $\alpha^{l}$ by $T_{\alpha}$; and $x$ such that $u_{\alpha, n}(x)=\alpha^{l+1}$ and whose height was lowered to $\alpha^{l}$ by $T_{\alpha}$. Therefore, by our induction hypothesis and by Pascal's identity,

$$
\begin{aligned}
\mid\{x & \left.\in[0,1]: u_{\alpha, n+1}(x)=\alpha^{l}\right\} \mid \\
& =\frac{1}{2} 4^{-n}\left(\begin{array}{c}
2 n \\
n+l
\end{array}\right)+\frac{1}{4} 4^{-n}\left(\begin{array}{c}
2 n \\
n+l-1
\end{array}\right)+\frac{1}{4} 4^{-n}\left(\begin{array}{c}
2 n \\
n+l+1
\end{array}\right) \\
& =4^{-n-1}\left[\left(\begin{array}{c}
2 n \\
n+l-1
\end{array}\right)+\left(\begin{array}{c}
2 n \\
n+l
\end{array}\right)\right]+4^{-n-1}\left[\left(\begin{array}{c}
2 n \\
n+l
\end{array}\right)+\left(\begin{array}{c}
2 n \\
n+l+1
\end{array}\right)\right] \\
& =4^{-n-1}\left[\left(\begin{array}{c}
2 n+1 \\
n+l
\end{array}\right)+\left(\begin{array}{c}
2 n+1 \\
n+l+1
\end{array}\right)\right]=4^{-n-1}\left(\begin{array}{c}
2 n+2 \\
n+l+1
\end{array}\right) .
\end{aligned}
$$

Hence part (iii) holds for all $n$. 
To prove part (iv), by Lemma 5.6 it will suffice to show that if $I$ and $J$ are two adjacent dyadic subintervals of $[0,1],|I|=|J|$, and $I$ to the left of $J$, then for all $k$,

$$
\alpha^{-2} u_{\alpha, k}(J) \leq u_{\alpha, k}(I) \leq \alpha^{2} u_{\alpha, k}(J) .
$$

We will show inequality (5.1) by induction. By inspection, it holds for $k=1$. Now suppose it holds for $k=n$. To show it holds for $k=n+1$ we consider three cases.

CASE 1: $I$ and $J$ are subintervals of $J_{i}^{n}$ for some $i$. On $J_{i}^{n}, u_{\alpha, n+1}=$ $a_{i, n+1} T_{\alpha}\left(\chi_{J_{i}^{n}}\right)$, so arguing as we did for $u_{\alpha, 1}$ we see that in this case (5.1) holds for $k=n+1$.

CASE 2: $I$ and $J$ are each the union of $2^{m}$ of the $J_{i}^{n}$ 's. Then by part (ii) above,

$$
u_{\alpha, n+1}\left(J_{i}^{n}\right)=\gamma_{\alpha} u_{\alpha, n}\left(J_{i}^{n}\right),
$$

and so it is immediate that in this case (5.1) holds for $k=n+1$.

CASe 3: $I \subset J_{i}^{n}, J \subset J_{i+1}^{n}$ for some $i$. There are two possibilities. If $|I| \leq\left|J_{i}^{n}\right| / 4$, then $u_{\alpha, n+1}(I)=u_{\alpha, n}(I)$ and $u_{\alpha, n+1}(J)=u_{\alpha, n}(J)$, so $(5.1)$ holds for $k=n+1$ in this case.

If $|I|=\left|J_{i}\right| / 2$ then a direct computation shows that

$$
u_{\alpha, n+1}(I)=\frac{\alpha^{-1}+1}{2} a_{i, n}|I|, \quad u_{\alpha, n+1}(J)=\frac{\alpha+1}{2} a_{i+1, n}|J| .
$$

Hence

$$
u_{\alpha, n+1}(I)=\frac{\alpha^{-1}+1}{\alpha+1} \cdot \frac{a_{i}}{a_{i+1}} u_{\alpha, n+1}(J)=\frac{\alpha^{-1}+1}{\alpha+1} \cdot \frac{u_{\alpha, n}\left(J_{i}^{n}\right)}{u_{\alpha, n}\left(J_{i+1}^{n}\right)} u_{\alpha, n+1}(J) .
$$

Inequality (5.1) would follow in this case for $k=n+1$ if

$$
\alpha^{-1} u_{\alpha, n}\left(J_{i+1}^{n}\right) \leq u_{\alpha, n}\left(J_{i}^{n}\right) \leq \alpha^{2} u_{\alpha, n}\left(J_{i+1}^{n}\right) .
$$

This inequality also follows by induction. It is immediate if $n=1$. To complete the induction, if it is true for some $n$ then, given $J_{i}^{n+1}$ and $J_{i+1}^{n+1}$ there are two cases: for some $j$ either they are both subintervals of some $J_{j}^{n}$ or $J_{i}^{n+1}=J_{j, 4}^{n}$ and $J_{i+1}^{n+1}=J_{j+1,1}^{n}$. In the first case we argue as we did when $n=1$. In the second case we note that $u_{\alpha, n+1}\left(J_{i}^{n+1}\right)=u_{\alpha, n}\left(J_{i}^{n+1}\right)$ and $u_{\alpha, n+1}\left(J_{i+1}^{n+1}\right)=u_{\alpha, n}\left(J_{i+1}^{n+1}\right)$. In either case we see that inequality (5.2) holds with $n$ replaced by $n+1$. Hence it is true for all $n$, and this completes the proof of part (iv).

Finally, to prove part (v), by Lemma 5.7 it will suffice to construct an increasing sequence $\left\{n_{k}\right\}$ and a sequence of sets $E_{k} \subset Q=[0,1]$ such that 
for any $q>1$,

$$
\lim _{k \rightarrow \infty} \frac{u_{\alpha, n_{k}}\left(E_{k}\right)}{u_{\alpha, n_{k}}(Q)}\left(\frac{\left|E_{k}\right|}{|Q|}\right)^{-q}=0 .
$$

Let $n_{k}=k^{2}$ and let $E_{k}=\left\{x \in[0,1]: u_{\alpha, n_{k}}(x)=\alpha^{-k}\right\}$. Then by part (iii) above,

$$
\left|E_{k}\right|=4^{-k^{2}}\left(\begin{array}{c}
2 k^{2} \\
k^{2}-k
\end{array}\right), \quad u_{\alpha, n_{k}}\left(E_{k}\right)=\alpha^{-k}\left|E_{k}\right| .
$$

We estimate $\left|E_{k}\right|$ using Stirling's formula:

$$
\begin{aligned}
\left|E_{k}\right| & =\frac{\left(2 k^{2}\right) !}{4^{k^{2}}\left(k^{2}-k\right) !\left(k^{2}+k\right) !} \approx \frac{\left(k^{2}\right)^{2 k^{2}}}{\left(k^{2}-k\right)^{k^{2}-k}\left(k^{2}+k\right)^{k^{2}+k}\left(k^{2}-1\right)^{1 / 2}} \\
& \approx\left(\frac{k^{4}}{k^{4}-k^{2}}\right)^{k^{2}}\left(\frac{k^{2}-k}{k^{2}+k}\right)^{k} \frac{1}{k} \\
& \approx\left(1+\frac{1}{k^{2}-1}\right)^{k^{2}-1}\left(1-\frac{2}{k+1}\right)^{k+1} \frac{1}{k} \approx \frac{1}{e k} .
\end{aligned}
$$

By part (ii) above, $u_{\alpha, n_{k}}(Q)=\gamma_{\alpha}^{k^{2}}$. Since $\gamma_{\alpha}>1$, for any $q>1$,

$$
\frac{u_{\alpha, n_{k}}\left(E_{k}\right)}{u_{\alpha, n_{k}}(Q)}\left(\frac{|Q|}{\left|E_{k}\right|}\right)^{q} \leq C \alpha^{-k} \gamma_{\alpha}^{-k^{2}} k^{q-1},
$$

and (5.3) follows at once.

REMARK. Since the doubling condition and the $A_{\infty}$ condition are not affected by multiplication by constants, parts (iv) and (v) of Theorem 5.5 remain true if we replace $u_{\alpha, n}$ by $\gamma_{\alpha}^{-n} u_{\alpha, n}$.

Construction of Example 5.2. It will suffice to construct $v$ on $[0, \infty)$, since by Lemma 5.6 , if $v$ is a doubling weight on $[0, \infty)$ and we extend it to $\mathbb{R}$ as an even function then it is doubling on $\mathbb{R}$.

Fix $\alpha>1$, say $\alpha=2$, and for $x \geq 0$ define

$$
v(x)=\sum_{n=0}^{\infty} \gamma_{\alpha}^{-n} u_{\alpha, n}(x-n) \chi_{[n, n+1)}(x) .
$$

By Theorem 5.5(ii), for any $n \geq 0$,

$$
\int_{n}^{n+1} v d x=1
$$

To show that $v$ is doubling on $[0, \infty)$ we will apply Lemma 5.6. By (5.4), if $I$ and $J$ are adjacent dyadic subintervals of $[0, \infty),|I|=|J| \geq 1$, then $v(I)=v(J)$. Further, by Theorem 5.5, if $I$ and $J$ are both subintervals of $[n, n+1]$ for some $n$ then $v(I) \leq \alpha^{2} v(J)$. Therefore, it remains to check 
that this is the case for two intervals $I$ and $J$ such that $I \subset[n-1, n]$ and $J \subset[n, n+1]$. Let $I^{\prime}=I-(n-1), J^{\prime}=J-n$. Fix $k \geq 1$ such that $4^{-k} \leq|I|<4^{-k+1}$. If $|I|=4^{-k}$ then $u_{\alpha, k}\left(I^{\prime}\right)=u_{\alpha, k}\left(J^{\prime}\right)=|I|$; if $|I|=$ $2 \cdot 4^{-k}$ then $u_{\alpha, k}\left(I^{\prime}\right)=\left(1+\alpha^{-1}\right)|I| / 2, u_{\alpha, k}\left(J^{\prime}\right)=(1+\alpha)|I| / 2$. Further, by Theorem 5.5(ii), $u_{\alpha, n-1}\left(I^{\prime}\right)=\gamma_{\alpha}^{n-1-k} u_{\alpha, k}\left(I^{\prime}\right)$ and $u_{\alpha, n}\left(J^{\prime}\right)=\gamma_{\alpha}^{n-k} u_{\alpha, k}\left(J^{\prime}\right)$. Therefore, $v(I)=\gamma_{\alpha}^{-k} u_{\alpha, k}\left(I^{\prime}\right)$ and $v(J)=\gamma_{\alpha}^{-k} u_{\alpha, k}\left(J^{\prime}\right)$, so

$$
\frac{1+\alpha^{-1}}{1+\alpha} v(J) \leq v(I) \leq v(J)
$$

Therefore, by Lemma 5.6, $v$ is a doubling weight.

Now fix $r \neq 1$. To see that $v^{r}$ is not a doubling weight, note that by Theorem 5.5(i),

$$
v(x)^{r}=\sum_{n=0}^{\infty} \gamma_{\alpha}^{-r n} u_{\alpha^{r}, n}(x-n) \chi_{[n, n+1)}(x) .
$$

Hence by Theorem 5.5(ii),

$$
\int_{n}^{n+1} v^{r} d x=\gamma_{\alpha^{r}}^{n} / \gamma_{\alpha}^{n r}
$$

Let $\Gamma_{r}=\gamma_{\alpha^{r}} / \gamma_{\alpha}^{r}$; if $r<1$ then $\Gamma_{r}<1$; if $r>1$ then $\Gamma_{r}>1$. Now let $I=[0, n]$ and $J=[n, 2 n]$. Then

$$
v^{r}(I)=\sum_{k=0}^{n-1} \Gamma_{r}^{k}=\frac{\Gamma_{r}^{n}-1}{\Gamma_{r}-1} \quad \text { and } \quad v^{r}(J)=\sum_{k=n}^{2 n-1} \Gamma_{r}^{k}=\frac{\Gamma_{r}^{2 n}-1}{\Gamma_{r}-1}-\frac{\Gamma_{r}^{n}-1}{\Gamma_{r}-1} .
$$

Therefore, if $r \neq 1$, then $v^{r}(I) / v^{r}(J)$ tends to either 0 or infinity as $n$ tends to infinity, so $v^{r}$ is not a doubling weight.

Construction of Example 5.3. As in the construction of Example 5.2, we will construct $w$ on $[0, \infty)$ and use Lemma 5.6 to show $w^{r}$ is a doubling weight for all $r>0$.

Again fix $\alpha>1$, say $\alpha=2$. Define the sequence $\left\{n_{k}\right\}$ as follows: let $n_{0}=0$, and for $k \geq 1$, if $2^{n} \leq k<2^{n+1}, n$ an integer, let $n_{k}=n$. Now define

$$
w(x)=\sum_{k=0}^{\infty} \gamma_{\alpha}^{-n_{k}} u_{\alpha, n_{k}}(x-k) \chi_{[k, k+1)}(x) .
$$

To show that $w^{r}$ is doubling and satisfies condition (D2), first note that if $I$ and $J$ are adjacent dyadic intervals with $|I|=|J| \leq 1$, then arguing as we did in the first half of Example 5.2, we obtain $w^{r}(I) \leq \alpha^{2 r} w^{r}(J)$.

Now suppose that $|I|=|J|=2^{n}, n \geq 1$, and $I$ is to the left of $J$. Given any $k$, arguing as in the second half of Example 5.2 gives $w^{r}([k, k+1])=\Gamma_{r}^{n_{k}}$. Let $I=\left[m 2^{n},(m+1) 2^{n}\right]$. There are three cases. If $m \geq 2$ then by our choice 
of the $n_{k}$ 's, $w^{r}(I)=w^{r}(J)$. If $m=1$ then $w^{r}(I)=\left(2 \Gamma_{r}\right)^{n}=\Gamma_{r} w^{r}(J)$. If $m=0$, then

$$
w^{r}(I)=1+\sum_{k=0}^{n-1} 2^{k} \Gamma_{r}^{k}=1+\frac{\left(2 \Gamma_{r}\right)^{n}-1}{2 \Gamma_{r}-1} \quad \text { and } \quad w^{r}(J)=\left(2 \Gamma_{r}\right)^{n},
$$

so

$$
\frac{w^{r}(I)}{w^{r}(J)}=\frac{1}{2 \Gamma_{r}-1}+\frac{1}{\left(2 \Gamma_{r}\right)^{n}}-\frac{1}{\left(2 \Gamma_{r}\right)^{n}\left(2 \Gamma_{r}-1\right)} .
$$

Therefore, we can conclude that for all $r>0, w^{r}$ is a doubling weight. Further, if $r \leq 1$ there exists a constant $R_{\alpha}$ such that $1 / 2<R_{\alpha} \leq \Gamma_{r} \leq 1$. (E.g. if $\alpha=2, R_{\alpha} \approx 0.97$.) Hence if $r \geq 1, D\left(w^{r}\right)$ is uniformly bounded.

Finally, by Theorem $5.5(\mathrm{v})$, since $n_{k}$ tends to infinity as $k$ tends to infinity, $w^{r}$ is not in $A_{\infty}$ for any $r$.

REMARK. The bound given by Lemma 5.6 for $D\left(w^{r}\right)$ is extremely poor, but we have been unable to estimate it (or more precisely, $D\left(u_{\alpha, n}\right)$ ) in any other way. For this reason we cannot show that $w$ satisfies condition (D3).

Doubling not necessary. We conclude this section with an example to show that doubling conditions are not necessary for the $W_{p}$ condition,

$$
\frac{1}{|I|} \int_{I} u d x \leq C_{1}\left(\frac{1}{|I|} \int_{I} \sigma d x\right)^{p+1},
$$

to govern the weighted norm inequalities for the minimal operator in higher dimensions.

EXAMPLE 5.8. There exists a pair of weights $(u, v) \in W_{p}\left(\mathbb{R}^{n}\right)$ such that neither $u$ or $\sigma=v^{1 /(p+1)}$ is doubling, but

$$
\int_{\mathbb{R}^{n}} \frac{u}{(m f)^{p}} d x \leq C \int_{\mathbb{R}^{n}} \frac{v}{|f|^{p}} d x
$$

for every $f$ such that $1 / f \in L^{p}(v)$.

Proof. For clarity we will construct our example in $\mathbb{R}^{2}$. The same argument extends to $\mathbb{R}^{n}, n \geq 3$.

Let $v_{0}(t)=e^{-1 /|t|}$. Then $v_{0}^{r}$ is not doubling for any $r>0$. Further, in [8] it was shown that if $u_{0}(t)$ is defined by the integral equation

$$
\frac{1}{t} \int_{0}^{t} u_{0} d s=\left(\frac{1}{t} \int_{0}^{t} v_{0}^{1 /(p+1)} d s\right)^{p+1}
$$

then $u_{0}$ is also not a doubling weight but $\left(u_{0}, v_{0}\right) \in W_{p}(\mathbb{R})$. We now define the pair $(u, v)$ by $u(x, y)=u_{0}(x) u_{0}(y), v(x, y)=v_{0}(x) v_{0}(y)$. It follows immediately from Fubini's theorem that $(u, v) \in W_{p}\left(\mathbb{R}^{2}\right)$ and that neither 
$u$ or $\sigma=v^{1 /(p+1)}$ is doubling. The same argument also shows that the pair $(u, v)$ does not satisfy the strong $W_{p}$ condition (1.2).

It therefore remains to show that inequality (5.5) holds. Define the minimal operator restricted to the first co-ordinate as follows:

$$
m_{1} f(x, y)=\inf _{I} \frac{1}{|I|} \int_{I}|f(t, y)| d t
$$

where the infimum is taken over all intervals in $\mathbb{R}$ which contain $x$. We define $m_{2}$, the minimal operator restricted to the second co-ordinate, similarly. Then, again by Fubini's theorem, $m f(x, y) \geq m_{1}\left(m_{2} f(x, y)\right)$, and inequality (5.5) follows by applying Theorem 1.2 to each variable in turn.

6. The centered minimal operator. In this section we prove Theorem 1.7. Throughout this section, let $\sigma=v^{1 /(p+1)}$.

The proof requires two covering lemmas. The first is a Whitney-type decomposition of a cube. This result is a special case of a covering lemma given by Sawyer [19]; because this case is much simpler we sketch the proof as a convenience to the reader.

Lemma 6.1. Given an open cube $Q$, there exists a sequence $\left\{Q_{k}\right\}$ of closed cubes contained in $Q$ such that: the $Q_{k}$ 's have disjoint interiors; $l\left(Q_{k}\right)=$ $\operatorname{dist}\left(Q_{k}, \partial Q\right) ; \bigcup_{k} Q_{k}=Q ; 2 Q_{k} \subset Q$ and any point in $Q$ is contained in at most $3 \cdot 2^{n-1}$ of the $2 Q_{k}$ 's.

Proof. Let $Q_{0}=\frac{1}{3} Q$. For $j \geq 1$, form the "shell"

$$
S_{j}=\left(3-2^{-j+1}\right) Q_{0} \backslash\left(3-2^{-j+2}\right) Q_{0} .
$$

Each shell $S_{j}$ can be divided into $m_{j}=2^{j n}\left(3-2^{-j+1}\right)^{n}-2^{j n}\left(3-2^{-j+2}\right)^{n}$ closed cubes with disjoint interiors. Further, each of these cubes $Q_{k}$ is such that $l\left(Q_{k}\right)=\operatorname{dist}\left(Q_{k}, \partial Q\right)=2^{-j}\left|Q_{0}\right|$. Clearly $Q$ is the union of the $Q_{k}$ 's, $k \geq 0$, and $2 Q_{k} \subset Q$. Finally, to see that the $2 Q_{k}$ 's have finite overlap, let $S_{0}=Q_{0}$ and $S_{-1}=\emptyset$. Then, if $x \in S_{j}, j \geq 0$, we have $x \in 2 Q_{k}$ for at most $2^{n-1}$ cubes $Q_{k}$ in each of $S_{j-1}, S_{j}$ and $S_{j+1}$.

The second covering lemma is a variant of the Besicovitch-Morse covering lemma, which we prove in the next two lemmas. (Our proof is adapted from the proof of the Besicovitch-Morse covering lemma given by Wheeden and Zygmund [24].)

LEMMA 6.2. Let $\left\{Q_{k}\right\}$ be a sequence of cubes such that for any $k>j$, $\left|Q_{k}\right| \leq \frac{3}{2}\left|Q_{j}\right|$ and $\frac{1}{5} Q_{k} \backslash Q_{j}$ is non-empty. Then there exists a constant $C_{n}$, depending only on the dimension $n$, such that every point $x \in \mathbb{R}^{n}$ is contained in at most $C_{n}$ of the $Q_{k}$ 's.

Proof. We will determine the degree of overlap of the $Q_{k}$ 's only at the origin; by translation the same value will hold for every point in $\mathbb{R}^{n}$. Let $\left\{Q_{k_{i}}\right\}$, 
$k_{i}<k_{i+1}$, be the collection of cubes which contain the origin and whose centers lie in the "first" quadrant - the quadrant where the co-ordinates of points are all non-negative. Let $h_{i}=l\left(Q_{k_{i}}\right)$. Then $Q_{k_{1}}$ must contain the cube $\left[0, h_{1} / 2\right)^{n}$. Since $\left|Q_{k_{i}}\right| \leq \frac{3}{2}\left|Q_{k_{1}}\right|$, for all $i>1$, we must have $h_{i} \leq(3 / 2)^{1 / n} h_{1} \leq \frac{5}{4} h_{1}$. Therefore the center of $Q_{k_{i}}, i>1$, must lie in the cube $\left[0, \frac{5}{8} h_{1}\right)^{n}$. Further, because $\frac{1}{5} Q_{k_{i}}$ cannot be contained in $Q_{k_{1}}$, the center of $Q_{k_{i}}$ cannot be in the cube $\left[0, \frac{3}{8} h_{1}\right)^{n}$. To see this, consider the limiting case: $h_{i}=\frac{5}{4} h_{1}$ and $\frac{1}{5} Q_{k_{i}}$ is just contained in $Q_{k_{1}}$. Then the center of $Q_{k_{i}}$ must lie within $h_{1} / 8$ of the edge of $Q_{k_{1}}$.

Therefore, the centers of the cubes $Q_{k_{i}}, i>1$, lie in $\left[0, \frac{5}{8} h_{1}\right)^{n} \backslash\left[0, \frac{3}{8} h_{1}\right)^{n}$. If a cube $Q_{k_{i}}$ has its center in this region, then, since it contains the origin, $\frac{3}{4} h_{1} \leq h_{i} \leq \frac{5}{4} h_{1}$. If two cubes $Q_{k_{i}}$ and $Q_{k_{j}}, j>i$, both have their centers in this region then their centers must be at least $h_{1} / 4$ apart. Otherwise, reasoning as we did before, we would have $\frac{1}{5} Q_{k_{j}} \subset Q_{k_{i}}$, a contradiction. There can be only a finite number, $C_{n}$, of such points, and $C_{n}$ depends only on the dimension. If we rescale so that $h_{1}=8$, then we get a rough estimate for $C_{n}$ as follows: count the number of unit cubes with integer co-ordinates which lie in the cube $[0,5]^{n}$. Since each such cube can contain the center of at most one $Q_{k_{i}}$, there are $5^{n}$ such cubes. If we repeat this argument for the cubes whose centers lie in the other $2^{n-1}$ quadrants, we see that there can be at most $10^{n}$ cubes containing the origin.

Remarks. (i) The constants $1 / 5$ and $3 / 2$ in Lemma 6.2 are not the only ones possible. We can replace them by any two positive constants $\delta<1$ and $\gamma>1$ such that $1 / 3>\delta \gamma^{1 / n}$.

(ii) The problem of finding the best value for the constant $C_{n}$ is closely related to an open problem in finite point sets. See Croft, Falconer and Guy [2, p. 154].

Lemma 6.3. Let $\left\{Q_{\alpha}\right\}$ be a collection of cubes in $\mathbb{R}^{n}$ whose union has finite measure, and for each $\alpha$ let $P_{\alpha}=\frac{1}{5} Q_{\alpha}$. Then there exists a sequence $\left\{Q_{k}\right\}$ of $Q_{\alpha}$ 's such that for each $\alpha, P_{\alpha} \subset Q_{k}$ for some $Q_{k}$, and such that each point $x \in \mathbb{R}^{n}$ is contained in at most $C_{n}$ of the $Q_{k}$ 's, where $C_{n}$ is the constant from Lemma 6.2.

Proof. Let $F_{0}=\emptyset$ and define

$$
\beta_{1}=\sup \left\{\left|Q_{\alpha}\right|: P_{\alpha} \notin F_{0}\right\} .
$$

Since $\bigcup_{\alpha} Q_{\alpha}$ has finite measure, $\beta_{1}<\infty$. Fix a cube $Q_{1}$ among the $Q_{\alpha}$ 's such that $\left|Q_{1}\right|>\frac{2}{3} \beta_{1}$. Define $F_{1}=\left\{P_{\alpha} \subset Q_{1}\right\}$ and

$$
\beta_{2}=\sup \left\{\left|Q_{\alpha}\right|: P_{\alpha} \notin F_{1}\right\} .
$$

Clearly $\beta_{2} \leq \beta_{1}$. If $\beta_{2}=0$ we are done. If not, continue this process. At the $k$ th stage, if $\beta_{k}>0$ fix a cube $Q_{k}$ among those used to define $\beta_{k}$ such that 
$\left|Q_{k}\right|>\frac{2}{3} \beta_{k}$. define $F_{k}=\left\{P_{\alpha} \subset Q_{k}\right\}$ and

$$
\beta_{k+1}=\sup \left\{\left|Q_{\alpha}\right|: P_{\alpha} \notin F_{j}, 1 \leq j \leq k\right\} .
$$

Clearly the $\beta_{k}$ 's are decreasing, so if $k>j$, then $\left|Q_{k}\right| \leq \beta_{k} \leq b_{j} \leq \frac{3}{2}\left|Q_{j}\right|$. Further, by our choice of $Q_{k}, P_{k} \backslash Q_{j} \neq \emptyset$. Therefore, by Lemma 6.2, the $Q_{k}$ 's have finite overlap.

It remains to show that each $P_{\alpha}$ is contained in some $Q_{k}$. If $\beta_{k}=0$ for some $k$ then this is immediate. Therefore, suppose that there are an infinite number of $Q_{k}$ 's, and suppose to the contrary that there exists an $\alpha$ such that $P_{\alpha}$ is not contained in any $Q_{k}$. Then for all $k, P_{\alpha} \notin F_{k-1}$, so $\left|Q_{\alpha}\right| \leq \beta_{k}$. If we can show that $\beta_{k}$ tends to 0 then we would have a contradiction. Since $\frac{2}{3} \beta_{k} \leq\left|Q_{k}\right| \leq \beta_{k}$, it will suffice to show that $\left|Q_{k}\right|$ tends to 0 .

Suppose instead that there exists $\delta>0$ such that $\left|Q_{k}\right| \geq \delta$. Then since there are an infinite number of $Q_{k}$ 's, and they have finite overlap, we have

$$
\infty=\sum\left|Q_{k}\right| \leq C_{n}\left|\bigcup_{\alpha} Q_{k}\right|<\infty
$$

a contradiction. Hence $\left|Q_{k}\right|$ tends to zero and we are done.

Proof of Theorem 1.7. A standard argument shows that the strong-type inequality implies the weak-type inequality, and the arguments in Sections 2 and 4 show that the weak-type inequality implies the $W_{p, c}$ condition,

$$
\frac{1}{|Q|} \int_{Q} u d x \leq D_{1}\left(\frac{1}{|2 Q|} \int_{2 Q} \sigma d x\right)^{p+1},
$$

and the strong-type inequality implies the $W_{p, c}^{*}$ condition,

$$
\int_{Q} \frac{u}{m_{c}\left(\sigma / \chi_{Q}\right)^{p}} d x \leq D_{3} \int_{Q} \sigma d x
$$

Therefore, to complete the proof it will suffice to show that (6.1) implies (6.2), and (6.2) implies the strong-type norm inequality.

REMARK. We do not have a direct proof that the $W_{p, c}^{*}$ condition implies the $W_{p, c}$ condition. The argument in Section 3 fails in the same way that the proof that the $W_{\infty}^{*}$ condition implies the $W_{p}^{*}$ condition fails in the centered case.

To prove that (6.1) implies (6.2), fix a cube $Q$ and apply Lemma 6.1 to form the sequence $\left\{Q_{k}\right\}$. For each $Q_{k}$, let $P_{k}$ be the union of all the cubes contained in $Q$ whose centers are in $Q_{k}$. Since $\operatorname{dist}\left(Q_{k}, Q\right)=l\left(Q_{k}\right), P_{k}$ is a rectangle whose volume is at most $4 \cdot 5^{n-1}\left|Q_{k}\right|$. Let $G_{n}=4 \cdot 5^{n-1}$. 
We now argue as we did in Section 3. Fix a cube $Q_{k}$ and for each $t>0$ let $E_{t}=\left\{x \in Q_{k}: m_{c}\left(\sigma / \chi_{Q}\right)(x)<1 / t\right\}$. Then for $R>0$ to be fixed below,

$$
\int_{Q_{k}} \frac{u}{m_{c}\left(\sigma / \chi_{Q}\right)^{p}} d x=p \int_{0}^{R} t^{p-1} u\left(E_{t}\right) d t+p \int_{R}^{\infty} t^{p-1} u\left(E_{t}\right) d t .
$$

The first integral on the right-hand side is bounded by $u\left(Q_{k}\right) R^{p}$. To estimate the second, note that if $x \in E_{t}$ there exists a cube $Q_{x, k}^{t}$ centered at $x$ such that $2 Q_{x, k}^{t} \subset P_{k}$ and

$$
\frac{1}{\left|2 Q_{x, k}^{t}\right|} \int_{2 Q_{x, k}^{t}} \sigma d y<1 / t
$$

By Lemma 2.1 there exists a subcollection $\left\{Q_{j, k}^{t}\right\}$ which covers $E_{t}$ and has finite overlap. We now repeat the argument in Section 3. With the same notation, inequality (3.5) becomes

$$
\begin{aligned}
p \int_{R}^{\infty} t^{p-1} u\left(E_{t}\right) d t & \leq p D_{1} \int_{R}^{\infty} t^{-2} \sum_{j}\left|Q_{j, k}^{t}\right| d t \\
& \leq p D_{1} B_{n}\left|P_{k}\right| / R=p D_{1} B_{n} G_{n}\left|Q_{k}\right| / R .
\end{aligned}
$$

Therefore the left-hand side of (6.3) is dominated by $u\left(Q_{k}\right) R^{p}+p C\left|Q_{k}\right| / R$. Fix $R$ so that $R^{p}=\sigma\left(2 Q_{k}\right) / u\left(Q_{k}\right)$. By the $W_{p, c}$ condition, $\left|Q_{k}\right| / R \leq$ $C \sigma\left(2 Q_{k}\right)$, so

$$
\int_{Q_{k}} \frac{u}{m_{c}\left(\sigma / \chi_{Q}\right)^{p}} d x \leq C \sigma\left(2 Q_{k}\right) .
$$

Since the $Q_{k}$ 's are disjoint and the $2 Q_{k}$ 's have finite overlap, if we sum over $k$ we get (6.2).

To prove that the $W_{p, c}^{*}$ condition implies the strong-type norm inequality, we will adapt the proof in Section 4. Fix a function $f$; without loss of generality we may assume that $f$ is non-negative. Further, arguing as we did in Section 2, we may also assume that there exists a cube $P$ and $\varepsilon>0$ such that $f(x)>\varepsilon$ for all $x \in P$ and $1 / f$ has support on $P$.

Fix $\alpha>1$, and for each integer $k$ let $A_{k}=\left\{x: \alpha^{-k-1} \leq m_{c} f(x)<\alpha^{-k}\right\}$. For each $x \in A_{k}$ there exists a cube $Q_{x}^{k}$ contained in $P$ and centered at $x$ such that

$$
\alpha^{-(k+1)} \leq \frac{1}{\left|Q_{x}^{k}\right|} \int_{Q_{x}^{k}} f d y<\alpha^{-k} .
$$

Let $P_{x}^{k}=\frac{1}{5} Q_{x}^{k}$. By the continuity of the integral there exists a small cube $K_{x}^{k}$ centered at $x$ and contained in $P_{x}^{k}$ such that if $y \in K_{x}^{k}$ then

$$
\frac{1}{\left|P_{x}^{k}\right|} \int_{P_{x}^{k}} \sigma d x \geq 2^{-1 / p} m_{c}\left(\sigma / \chi_{P_{x}^{k}}\right)(y) .
$$


By Lemma 2.1 there exists a subcollection $\left\{K_{j}^{k}\right\}$ such that $A_{k} \subset \bigcup_{j} K_{j}^{k}$ and the $K_{j}^{k}$ 's have finite overlap. Let $E_{j}^{k}=A_{k} \cap K_{j}^{k}$. Then, since the $A_{k}$ 's are disjoint, the $E_{j}^{k}$ 's have finite overlap for all $j$ and $k$.

Since $f(x)>\varepsilon$ on $P$, we have $u\left(\left\{x: m_{c} f(x)=0\right\}\right)=0$. Therefore, we can proceed as we did in Section 4; using the same notation we get

$$
\int_{\mathbb{R}^{n}} \frac{u}{\left(m_{c} f\right)^{p}} d x \leq \alpha^{p} \int_{X} S(f / \sigma)^{p} d \omega \leq \alpha^{p} \int_{X} T\left((f / \sigma)^{-p / 2}\right)^{2} d \omega .
$$

As before, it will suffice to show that $T$ is weak $(1,1)$. For each $\lambda>0$, let

$$
E_{\lambda}=\{(j, k) \in X: T h(j, k)>\lambda\} .
$$

Then

$$
\begin{aligned}
\omega\left(E_{\lambda}\right) & =\sum_{(j, k) \in E_{\lambda}} u\left(E_{j}^{k}\right)\left(\frac{1}{\left|Q_{j}^{k}\right|} \int_{Q_{j}^{k}} \sigma d x\right)^{-p} \\
& \leq 5^{n p} \sum_{(j, k) \in E_{\lambda}} u\left(E_{j}^{k}\right)\left(\frac{1}{\left|P_{j}^{k}\right|} \int_{P_{j}^{k}} \sigma d x\right)^{-p} \\
& \leq 2 \cdot 5^{n p} \sum_{(j, k) \in E_{\lambda}} \int_{E_{j}^{k}} \frac{u}{m_{c}\left(\sigma / \chi_{P_{j}^{k}}\right)^{p}} d x
\end{aligned}
$$

By our choice of the $Q_{j}^{k}$ 's, their union is bounded and so has finite measure. Therefore, by Lemma 6.3 there exists a subcollection $\left\{Q_{n}\right\}$ with finite overlap such that each $P_{j}^{k}$ is contained in some $Q_{n}$. Since the $E_{j}^{k}$ 's have finite overlap, it follows that

$$
\begin{aligned}
2 \cdot 5^{n p} \sum_{(j, k) \in E_{\lambda}} \int_{E_{j}^{k}} \frac{u}{m_{c}\left(\sigma / \chi_{P_{j}^{k}}\right)^{p}} d x & \leq 2 \cdot 5^{n p} \sum_{n} \sum_{P_{j}^{k} \subset Q_{n}} \int_{E_{j}^{k}} \frac{u}{m_{c}\left(\sigma / \chi_{Q_{n}}\right)^{p}} d x \\
& \leq 2 B_{n} 5^{n p} \sum_{n} \int_{Q_{n}} \frac{u}{m_{c}\left(\sigma / \chi_{\left.Q_{n}\right)^{p}}\right.} d x \\
& \leq 2 B_{n} D_{3} 5^{n p} \sum_{n} \int_{Q_{n}} \sigma d x \\
& \leq \frac{2 B_{n} D_{3} 5^{n p}}{\lambda} \sum_{n} \int_{Q_{n}} h \sigma d x \\
& \leq \frac{2 B_{n} C_{n} D_{3} 5^{n p}}{\lambda} \int_{\mathbb{R}^{n}} h \sigma d x .
\end{aligned}
$$

Therefore $T$ is weak-type $(1,1)$ and our proof is complete.

Remark. The weak $(1,1)$ constant of $T$ is $2 B_{n} C_{n} D_{3} 5^{n p}$, and so the strong-type $(2,2)$ constant is $M=16 B_{n} C_{n} D_{3} 5^{n p}$. Hence $D_{4} \leq 8 M \alpha^{p}$. 
While we can take the limit as $\alpha$ tends to 1 to get $D_{4} \leq 8 M, M$ itself still depends on $p$.

The proof on $\mathbb{R}$. If we restrict ourselves to the real line we can modify our proof to get the strong-type $(2,2)$ constant for $T$ independent of $p$. The argument follows closely the proof of the main theorem in [4]; here we sketch the details.

Begin the proof as before, and choose the $Q_{x}^{k}$ 's to be open intervals. Omit the $P_{x}^{k}$ 's and choose each interval $K_{x}^{k}$ to be concentric with the interval $Q_{x}^{k}$ and such that if $y \in K_{x}^{k}$ then

$$
\frac{1}{\left|Q_{x}^{k}\right|} \int_{Q_{x}^{k}} \sigma d x \geq 2^{-1 / p} m_{c}\left(\sigma / \chi_{Q_{x}^{k}}\right)(y)
$$

Continue the above proof exactly the same until we have to show that $T$ is weak-type $(1,1)$. Here we estimate as follows:

$$
\omega\left(E_{\lambda}\right)=\sum_{(j, k) \in E_{\lambda}} u\left(E_{j}^{k}\right)\left(\frac{1}{\left|Q_{j}^{k}\right|} \int_{Q_{j}^{k}} \sigma d x\right)^{-p} \leq 2 \sum_{(j, k) \in E_{\lambda}} \int_{E_{j}^{k}} \frac{u}{m_{c}\left(\sigma / \chi_{Q_{j}^{k}}\right)^{p}} d x
$$

Let $G=\bigcup Q_{j}^{k}$. Since the $Q_{j}^{k}$ 's are open, $G$ is open and so it is the union of disjoint open intervals $Q_{n}$. Further, each $Q_{n}$ is the union of $Q_{j}^{k}$ 's, so each set $E_{j}^{k}$ is contained in a unique $Q_{n}$. Finally, by a lemma of Muckenhoupt [16] (also see [4]) we have

$$
\int_{Q_{n}} \sigma d x \leq \frac{2}{\lambda} \int_{Q_{n}} h \sigma d x
$$

Therefore, if we use this collection $\left\{Q_{n}\right\}$ the above argument goes through, and we conclude that the weak $(1,1)$ constant for $T$ in this case is $4 D_{3}$, so the strong $(2,2)$ constant is $32 D_{3}$, which does not depend on $p$.

The one-weight case. We conclude this section by proving the analogue of Theorem 1.1 for the centered minimal operator. For brevity, we write $w \in W_{p, c}$ instead of $(w, w) \in W_{p, c}$. Recall that a function $w$ satisfies the weak $A_{\infty}$ condition if, given any cube $Q$ and a measurable set $E \subset Q$, there exist constants $C$ and $\delta$ such that

$$
\frac{w(E)}{w(2 Q)} \leq C\left(\frac{|E|}{|Q|}\right)^{\delta}
$$

This condition was introduced by Sawyer [19] to generalize the good- $\lambda$ inequality of Coifman and Fefferman [1]. He proved that $w \in$ weak $A_{\infty}$ if and only if it satisfied the weak reverse Hölder inequality for some $s>1$ :

$$
\text { weak } R H_{s}: \quad\left(\frac{1}{|Q|} \int_{Q} w^{s} d x\right)^{1 / s} \leq C \frac{1}{|2 Q|} \int_{2 Q} w d x \text {. }
$$


The weak $R H_{s}$ condition plays an important role in the study of PDE's, potential theory and quasi-conformal mappings. (See, for example, Giaquinta [12], Iwaniec and Nolder [15] and Stredulinsky [21].) It has many properties in common with the reverse Hölder inequality; here we need two.

Lemma 6.4. If $w \in$ weak $R H_{s}$ for some $s>1$ then:

(i) there exists $t>s$ such that $w \in$ weak $R H_{t}$;

(ii) for each $r, 0<r<1, w^{r} \in$ weak $R H_{s / r}$.

The proof of (i) is found in [12] and [21]; the proof of (ii) is in [15] and Heinonen, Kilpeläinen and Martio [14, pp. 66-68].

We can now prove our result.

TheOREM 6.5. Given a weight $w$ and $p, 0<p<\infty$, the following are equivalent:

(i) $w \in W_{p, c}$;

(ii) $w \in$ weak $A_{\infty}$.

Proof. Suppose $w \in W_{p, c}$; then $w^{1 /(p+1)} \in$ weak $R H_{p+1}$, so by Lemma 6.4, there exists $t>p+1$ such that $w^{1 /(p+1)} \in$ weak $R H_{t}$. Therefore, by Hölder's inequality, $w \in$ weak $R H_{t /(p+1)}$, so $w \in$ weak $A_{\infty}$.

Now suppose that $w \in$ weak $A_{\infty}$. Then for some $s>1, w \in$ weak $R H_{s}$, so by Lemma 6.4 , for each $p>0, w^{1 /(p+1)} \in$ weak $R H_{s(p+1)}$, which implies that $w \in W_{p, c}$.

7. The equality $M_{0} f(x)=M_{0}^{*} f(x)$. In this section we prove Theorem 1.8. The proof uses two lemmas from [6]. Recall that $v \in I_{\infty}$ if

$$
\limsup _{Q, \sigma} \frac{1}{|Q|}\left(\frac{1}{|Q|} \int_{Q} v^{-\sigma} d x\right)^{1 / \sigma}<\infty,
$$

where the upper limit is taken over all cubes $Q$ containing the origin and all $\sigma>0$ as $|Q|$ tends to infinity and $\sigma$ tends to 0 .

LEMMA 7.1. Let $Q_{0}$ be a cube (possibly infinite) and suppose that $\operatorname{supp} f$ $=Q_{0}$. If for some $p, 0<p<\infty, f \in L^{p}\left(Q_{0}\right)$ and $\log f \in L_{\mathrm{loc}}^{1}\left(Q_{0}\right)$ then $M_{0}^{*} f(x)=M_{0} f(x)$ for almost every $x$.

Lemma 7.2. Let $v \in I_{\infty}$ and suppose that for some $p, 0<p<\infty$, $f \in L^{p}(v)$. Let $Q_{k}$ be the cube centered at the origin such that $l\left(Q_{k}\right)=k$. Then the following are true:

(i) for all $x, M_{0}^{*} f(x)=\lim _{k \rightarrow \infty} M_{0}^{*}\left(f \chi_{Q_{k}}\right)(x)$;

(ii) there exists $r>0$ such that $f \in L_{\mathrm{loc}}^{r}$. 
Remarks. (i) In [6, Corollary 2.2], it was implicitly assumed that the cube $Q_{0}$ in Lemma 7.1 was finite; however the same argument works for all cubes.

(ii) In [6, Lemma 4.3], Lemma 7.2 is only proved for $p=1$, but the proof adapts immediately to the case of arbitrary $p$. Further, (ii) is actually part of the proof of (i).

Proof of Theorem 1.8. Fix a function $f$ such that $\log |f|$ is locally integrable and $f \in L^{p}(v)$ for some $p>0$. Without loss of generality we may assume that $f$ is non-negative. Then Lemmas 7.1 and 7.2 together imply that for almost every $x$,

$$
M_{0}^{*} f(x)=\lim _{k \rightarrow \infty} M_{0}^{*}\left(f \chi_{Q_{k}}\right)(x)=\lim _{k \rightarrow \infty} M_{0}\left(f \chi_{Q_{k}}\right)(x) .
$$

Since $M_{0}\left(f \chi_{Q_{k}}\right)(x) \leq M_{0} f(x)$, to show that the right-hand side equals $M_{0} f(x)$, it will suffice to show that

$$
M_{0} f(x) \leq \lim _{k \rightarrow \infty} M_{0}\left(f \chi_{Q_{k}}\right)(x) .
$$

Fix $\varepsilon>0$. Then there exists a cube $Q$ containing $x$ such that

$$
M_{0} f(x)-\varepsilon<\exp \left(\frac{1}{|Q|} \int_{Q} \log f d x\right) .
$$

But for all $k$ sufficiently large, $Q \subset Q_{k}$, so the right-hand side is dominated by $M_{0}\left(f \chi_{Q_{k}}\right)(x)$. Therefore,

$$
M_{0} f(x)-\varepsilon \leq \lim _{k \rightarrow \infty} M_{0}\left(f \chi_{Q_{k}}\right)(x),
$$

and since $\varepsilon>0$ is arbitrary, inequality (7.1) follows at once.

To complete the proof we will construct a non-negative function $f$ on $\mathbb{R}$ such that $f$ is bounded, supp $f=[0,1], \log f \in L^{1}[0,1]$ and $M_{0}^{*} f(0)>$ $M_{0} f(0)$.

For $n>1$, let $I_{n}=\left[1 / n-1 / n^{4}, 1 / n\right]$ and let $I=\bigcup_{n} I_{n}$. Define

$$
f(x)= \begin{cases}e^{-n^{2}}, & x \in I_{n}, \\ 1, & x \in[0,1] \backslash I, \\ 0, & x \in \mathbb{R} \backslash[0,1] .\end{cases}
$$

Clearly $f$ is bounded and $\operatorname{supp} f=[0,1]$. (Hence $f \in L^{1}(v)$ for any locally integrable $v$.) Second,

$$
\int_{0}^{1}|\log f| d x=\sum_{n=2}^{\infty} n^{2}\left|I_{n}\right|=\sum_{n=2}^{\infty} n^{-2}<\infty .
$$

Third, fix $r, 0<r<1$. Then by a standard calculus argument, for any 
$n>1$, we have

$$
\begin{aligned}
M_{r} f(0) & \geq\left(n \int_{0}^{1 / n} f^{r} d x\right)^{1 / r} \geq(n|[0,1 / n] \backslash I|)^{1 / r} \\
& \geq\left(n\left(\frac{1}{n}-\sum_{k \geq n} \frac{1}{k^{4}}\right)\right)^{1 / r} \geq\left(n\left(\frac{1}{n}-\frac{1}{3 n^{3}}\right)\right)^{1 / r} \\
& =\left(1-\frac{1}{3 n^{2}}\right)^{1 / r} .
\end{aligned}
$$

The last term tends to 1 as $n$ tends to infinity. Therefore $M_{r} f(0)=1$ for all $r$, so $M_{0}^{*} f(0)=1$.

Finally, fix $t, 0<t \leq 1$. For some $n \geq 1,(n+1)^{-1}<t \leq n^{-1}$. Then arguing as before, we obtain

$$
\frac{1}{t} \int_{0}^{t} \log f d x \leq \frac{-1}{t} \sum_{k \geq n+1} k^{2}\left|I_{k}\right| \leq-n \sum_{k \geq n+1} k^{-2} \leq \frac{-n}{n}=-1 .
$$

It follows that $M_{0} f(0) \leq e^{-1}<1=M_{0}^{*} f(0)$.

Remark. Wik [26] established the equality of $M_{0} f(x)$ and $M_{0}^{*} f(x), f$ non-negative, given the following conditions:

(i) for some $r>0, f \in L_{\mathrm{loc}}^{r}$;

(ii) $\log f \in L_{\text {loc }}^{1}$;

(iii) for all $t>0,|\{x: f(x)>t\}|<\infty$;

(iv) $\lim \sup _{k \rightarrow \infty}\left|Q_{k}\right|^{-1} \int_{Q_{k}} \log ^{+} f d x<\infty$, where $Q_{k}$ is the cube centered at the origin with $l\left(Q_{k}\right)=k$.

Theorem 1.8 is a generalization of this result. To see this, first note that condition (ii) is a shared hypothesis. Second, we claim that conditions (i) and (iv) follow from the assumption that there exists $v \in I_{\infty}$ such that $f \in L^{p}(v)$ for some $p>0$. Indeed, (i) follows from Lemma 7.2.

To show condition (iv): if $v \in I_{\infty}$, there exist constants $N$ and $M$ such that if $\sigma<1 / N$ and $\left|Q_{k}\right|>N$ then

$$
\frac{1}{\left|Q_{k}\right|}\left(\frac{1}{\left|Q_{k}\right|} \int_{Q_{k}} v^{-\sigma} d x\right)^{1 / \sigma}<M .
$$

Fix $k$ such that $\left|Q_{k}\right|>N$ and $r$ such that $0<r /(p-r)<1 / N$. Let $J_{k}=\left\{x \in Q_{k}: f(x) \geq 1\right\}$. Then $\log ^{+} f(x)=\log f(x) \chi_{J_{k}}(x)$ for $x \in Q_{k}$. Hence, by Jensen's inequality,

$$
\exp \left(\frac{1}{\left|J_{k}\right|} \int_{Q_{k}} \log ^{+} f d x\right)=\exp \left(\frac{1}{\left|J_{k}\right|} \int_{J_{k}} \log f d x\right) \leq\left(\frac{1}{\left|J_{k}\right|} \int_{J_{k}} f^{r} d x\right)^{1 / r} .
$$


Then by Hölder's inequality,

$$
\begin{aligned}
\frac{1}{\left|J_{k}\right|} \int_{J_{k}} f^{r} d x \leq & \frac{\left|Q_{k}\right|}{\left|J_{k}\right|}\left(\int_{\mathbb{R}^{n}} f^{p} v d x\right)^{r / p} \\
& \times\left(\frac{1}{\left|Q_{k}\right|}\left(\frac{1}{\left|Q_{k}\right|} \int_{Q_{k}} v^{-r /(p-r)} d x\right)^{(p-r) / r}\right)^{r / p} \\
\leq & \frac{\left|Q_{k}\right|}{\left|J_{k}\right|}\|f\|_{L^{p}(v)}^{r} M^{r}
\end{aligned}
$$

Therefore,

$$
\frac{1}{\left|Q_{k}\right|} \int_{Q_{k}} \log ^{+} f d x \leq \frac{\left|J_{k}\right|}{\left|Q_{k}\right|} \log \left(M\|f\|_{L^{p}(v)}\right)+r^{-1} \frac{\left|J_{k}\right|}{\left|Q_{k}\right|} \log \left(\frac{\left|Q_{k}\right|}{\left|J_{k}\right|}\right) .
$$

Since $0<\left|J_{k}\right| /\left|Q_{k}\right| \leq 1$ and the function $x \log (1 / x)$ is bounded on $(0,1]$, this establishes condition (iv).

Finally, we note that condition (iii) need not hold for $f \in L^{p}(v)$. We construct a weight $v$ on the real line as follows: for $n \geq 0$ let $I_{n}=\left[2^{n}-1,2^{n}\right]$. For $x \geq 0$ define

$$
v(x)= \begin{cases}4^{-n}, & x \in I_{n} \\ 1, & \text { otherwise. }\end{cases}
$$

Extend $v$ to $\mathbb{R}$ as an even function. A straightforward computation shows that $v \in I_{\infty}$. Now define a function $f$ by

$$
f(x)= \begin{cases}2^{n}, & |x| \in I_{n} \\ 1 / x^{2}, & \text { otherwise. }\end{cases}
$$

Then it is immediate that $f \in L^{1}(v)$, but

$$
|\{x: f(x)>1\}|=2 \sum_{n=1}^{\infty}\left|I_{n}\right|=\infty .
$$

A conjecture of Wik. We conclude this section by proving a conjecture made by Wik [26]. Given a weight $w \in A_{\infty}$, let

$$
\begin{aligned}
& m_{\infty}(w)=\sup \left\{\int_{\mathbb{R}^{n}} M_{0} f w d x: \int_{\mathbb{R}^{n}}|f| w d x=1\right\}, \\
& m_{\infty}^{*}(w)=\sup \left\{\int_{\mathbb{R}^{n}} M_{0}^{*} f w d x: \int_{\mathbb{R}^{n}}|f| w d x=1\right\} .
\end{aligned}
$$

Since $w \in A_{\infty}$, we have $w \in W_{\infty}^{*}$ and $w \in I_{\infty}$, so both supremums are finite. (See [6] for details.) Clearly $m_{\infty}(w) \leq m_{\infty}^{*}(w)$. Wik [26] showed that there exists a constant $C$ such that $m_{\infty}^{*}(w) \leq C m_{\infty}(w)$ and conjectured that in fact these two quantities are equal. Using Theorem 1.8 we show that this is true. 
Theorem 7.3. Given $w \in A_{\infty}, m_{\infty}(w)=m_{\infty}^{*}(w)$.

Proof. Fix $w \in A_{\infty}$ and fix a non-negative function $f \in L^{1}(w)$ such that $\int f w d x=1$. We first construct a sequence $\left\{f_{j}\right\}$ that decreases to $f$ and such that for each $j, f_{j} \in L^{1}(w)$ and $\log f_{j} \in L_{\text {loc }}^{1}$. For each $k \geq 1$, let $Q_{k}$ be the cube centered at the origin with $l\left(Q_{k}\right)=k$, and let $S_{k}=Q_{k} \backslash Q_{k-1}$. Choose a positive decreasing sequence $\left\{a_{k}\right\}$ such that

$$
\sum_{k=1}^{\infty} a_{k} \int_{S_{k}} w d x<\infty .
$$

For $j \geq 1$ and $x \in S_{k}$, define $f_{j}(x)=f(x)+a_{k} / j$. Clearly, $\left\{f_{j}\right\}$ decreases to $f$. Further, on each cube $Q_{k}$ the functions $f_{j}$ are bounded below, so $\log f_{j} \in L_{\mathrm{loc}}^{1}$. Finally, $f_{j} \in L^{1}(w)$, since

$$
\begin{aligned}
\int_{\mathbb{R}^{n}} f_{j} w d x & =\sum_{k=1}^{\infty} \int_{S_{k}} f_{j} w d x=\sum_{k=1}^{\infty} \int_{S_{k}}\left(f+a_{k} / j\right) w d x \\
& =\int_{\mathbb{R}^{n}} f w d x+\frac{1}{j} \sum_{k=1}^{\infty} a_{k} \int_{S_{k}} w d x<\infty .
\end{aligned}
$$

Since the $f_{j}$ 's are a decreasing sequence,

$$
M_{0}^{*} f(x) \leq \lim _{j \rightarrow \infty} M_{0}^{*} f_{j}(x) .
$$

Therefore, by Fatou's lemma and Theorem 1.8,

$$
\begin{aligned}
\int_{\mathbb{R}^{n}} M_{0}^{*} f w d x & \leq \lim _{j \rightarrow 0} \int_{\mathbb{R}^{n}} M_{0}^{*} f_{j} w d x=\lim _{j \rightarrow 0} \int_{\mathbb{R}^{n}} M_{0} f_{j} w d x \\
& =\lim _{j \rightarrow 0}\left\|f_{j}\right\|_{L^{1}(w)} \int_{\mathbb{R}^{n}} M_{0}\left(f_{j} /\left\|f_{j}\right\|_{L^{1}(w)}\right) w d x \\
& \leq m_{\infty}(w) \lim _{j \rightarrow 0}\left\|f_{j}\right\|_{L^{1}(w)}=m_{\infty}(w) .
\end{aligned}
$$

Since this is true for all such $f, m_{\infty}^{*}(w) \leq m_{\infty}(w)$ and we are done.

\section{References}

[1] R. Coifman and C. Fefferman, Weighted norm inequalities for maximal functions and singular integrals, Studia Math. 51 (1974), 241-250.

[2] H. Croft, K. Falconer and R. Guy, Unsolved Problems in Geometry, Problem Books in Math., Springer, New York, 1991.

[3] D. Cruz-Uribe, SFO, Piecewise monotonic doubling measures, Rocky Mountain J. Math. 26 (1996), 1-39.

[4] -, A new proof of the two weight norm inequality for the one-sided fractional maximal operator, Proc. Amer. Math. Soc. 125 (1997), 1419-1424. 
[5] D. Cruz-Uribe, SFO, and C. J. Neugebauer, The structure of the reverse Hölder classes, Trans. Amer. Math. Soc. 347 (1995), 2941-2960.

[6] - - - Weighted norm inequalities for the geometric maximal operator, Publ. Mat. 42 (1998), 239-263.

[7] - - - Weighted norm inequalities for the centered maximal operator on $\mathbb{R}^{+}$, Ricerche Mat. 49 (1999), 225-241.

[8] D. Cruz-Uribe, SFO, C. J. Neugebauer and V. Olesen, Norm inequalities for the minimal and maximal operator, and differentiation of the integral, Publ. Mat. 41 (1997), 577-604.

[9] - - - - , Weighted norm inequalities for a family of one-sided minimal operators, Illinois J. Math. 41 (1997), 77-92.

[10] C. Fefferman and B. Muckenhoupt, Two nonequivalent conditions for weight functions, Proc. Amer. Math. Soc. 45 (1974), 99-104.

[11] J. García-Cuerva and J. L. Rubio de Francia, Weighted Norm Inequalities and Related Topics, North-Holland Math. Stud. 116, North-Holland, Amsterdam, 1985.

[12] M. Giaquinta, Multiple Integrals in the Calculus of Variations and Nonlinear Elliptic Systems, Ann. of Math. Stud. 105, Princeton Univ. Press, Princeton, 1983.

[13] M. de Guzmán, Differentiation of Integrals in $\mathbb{R}^{n}$, Lecture Notes in Math. 481, Springer, New York, 1975.

[14] J. Heinonen, T. Kilpeläinen and O. Martio, Nonlinear Potential Theory of Degenerate Elliptic Equations, Clarendon Press, Oxford, 1993.

[15] T. Iwaniec and C. Nolder, Hardy-Littlewood inequality for quasiregular mappings in certain domains of $\mathbb{R}^{n}$, Ann. Acad. Sci. Fenn. Ser. A I Math. 10 (1985), 267-282.

[16] B. Muckenhoupt, Weighted norm inequalities for the Hardy maximal function, Trans. Amer. Math. Soc. 165 (1972), 207-226.

[17] C. Sadosky, Interpolation of Operators and Singular Integrals, Dekker, New York, 1979.

[18] E. T. Sawyer, A characterization of a two-weight norm inequality for maximal operators, Studia Math. 75 (1982), 1-11.

[19] —, Two weight norm inequalities for certain maximal and integral operators, in: Lecture Notes in Math. 908, Springer, New York, 1982, 102-127.

[20] P. Sjögren, A remark on the maximal function for measures on $\mathbb{R}^{n}$, Amer. J. Math. 105 (1983), 1231-1233.

[21] E. Stredulinsky, Weighted Inequalities and Degenerate Elliptic Partial Differential Equations, Lecture Notes in Math. 1074, Springer, Berlin, 1984.

[22] J. O. Strömberg, Non-equivalence between two kinds of conditions on weight functions, in: Harmonic Analysis in Euclidean Spaces, G. Weiss and S. Wainger (eds.), Proc. Sympos. Pure Math. 35, Part I, Amer. Math. Soc., Providence, 1979, 141-148.

[23] R. Wheeden, A characterization of some weighted norm inequalities for the fractional maximal function, Studia Math. 107 (1993), 257-272.

[24] R. Wheeden and A. Zygmund, Measure and Integral: An Introduction to Real Analysis, Dekker, New York, 1977.

[25] I. Wik, On Muckenhoupt's classes of weight functions, Studia Math. 94 (1989), 245-255.

[26] - , The geometric maximal function, preprint. 
[27] X. Yin and B. Muckenhoupt, Weighted inequalities for the maximal geometric mean operator, Proc. Amer. Math. Soc. 124 (1996), 75-81.

Department of Mathematics

Trinity College

Hartford, CT 06106-3100, U.S.A.

E-mail: david.cruzuribe@mail.trincoll.edu

Received December 28, 1998

Revised version September 8, 1999 